\title{
Solomon Islands: Tax Summary and Statistical Appendix
}

This Tax Summary and Statistical Appendix paper for the Solomon Islands was prepared by a staff team of the International Monetary Fund as background documentation for the periodic consultation with the member country. It is based on the information available at the time it was completed on September 10, 2008. The views expressed in this document are those of the staff team and do not necessarily reflect the views of the government of the Solomon Islands or the Executive Board of the IMF.

The policy of publication of staff reports and other documents by the IMF allows for the deletion of market-sensitive information.

Copies of this report are available to the public from

International Monetary Fund $\bullet$ Publication Services

700 19th Street, N.W. • Washington, D.C. 20431

Telephone: (202) 623-7430 • Telefax: (202) 623-7201

E-mail: publications@imf.org • Internet: http://www.imf.org

Price: $\$ 18.00$ a copy

\section{International Monetary Fund \\ Washington, D.C.}





\title{
INTERNATIONAL MONETARY FUND
}

\section{SOLOMON ISLANDS}

\section{Tax Summary and Statistical Appendix}

\author{
Prepared by Gilda Fernandez and Francis Vitek
}

Approved by Asia and Pacific Department

September 10, 2008

Contents

Page

Tax Summary 3

\section{Statistical Tables}

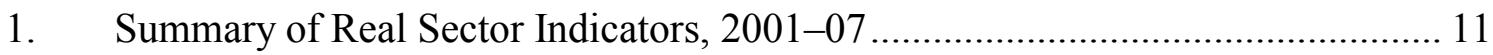

2. Real GDP Growth Rates by Sectoral Origin, 2004-07 ..................................... 12

3. Honiara Retail Price Index, 2004-08 ................................................................. 13

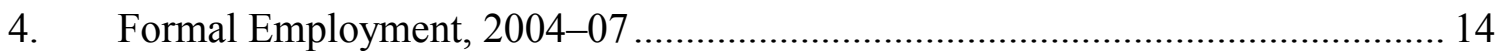

5. Central Government Operations, 2002-07........................................................ 15

6. Central Government Revenue and Grants, 2002-07 .......................................... 16

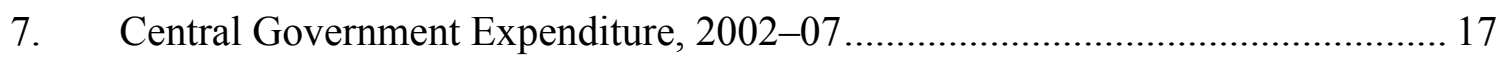

8. Central Government Budget Financing, 2002-07 .............................................. 18

9. Central Government Gross Domestic Debt, 2002-07 ......................................... 19

10. Equity Investment of the Investment Corporation of

Solomon Islands, 2003-07 .................................................................. 20

11. Summary Accounts of the Banking System, 2004-07 .................................... 21

12. Assets and Liabilities of the Central Bank of Solomon Islands, 2004-07 ........... 22

13. Assets and Liabilities of Commercial Banks, 2004-07 ....................................... 23

14a. Commercial Bank Advances and Loans, 2004-08 ............................................ 24

14b. Commercial Bank Advances and Loans, 2004-08 .......................................... 25

15. Commercial Bank Interest Rates on Savings and Time Deposits, 2004-08 ........ 26

16. Commercial Bank Interest Rates on Loans and Overdrafts, 2004-08.................. 27

17. Assets and Liabilities of the National Provident Fund, 2004-08 ........................ 28

18. Income Statement of the National Provident Fund, 2003-07 ............................. 29

19. Assets and Liabilities of the Development Bank of Solomon Islands, 2003-07 .. 30

20. Income Statement of the Development Bank of Solomon Islands, 2003-07 ....... 31

21. Balance of Payments, 2003-07 .................................................................... 32 


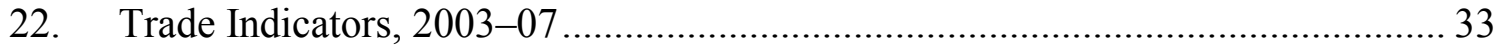

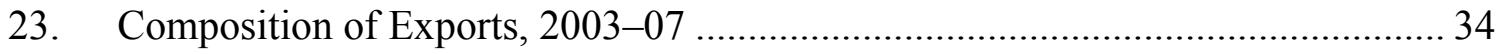

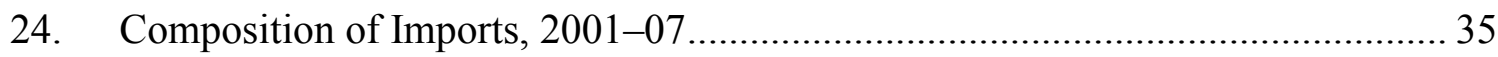

25. Exports by Country of Destination, 2001-07 ……................................................. 36

26. Imports by Country of Origin, 2001-07 ………….............................................. 37

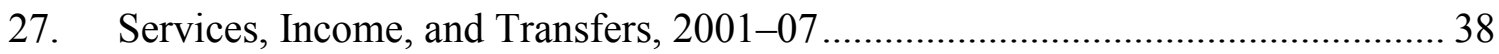

28. Medium- and Long-Term Government External Debt

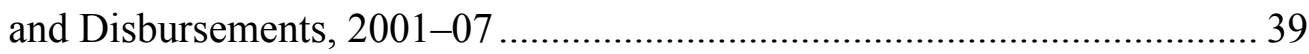

29. Medium- and Long-Term External Government

Debt-Service Payments, 2001-07 ……………........................................... 40

30. Medium- and Long-Term External Debt Indicators, 2001-07 .............................. 41

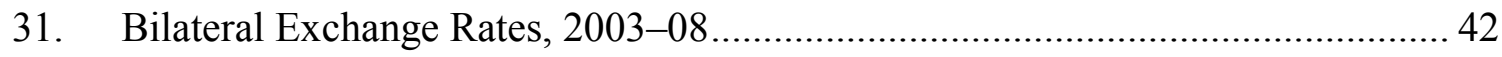

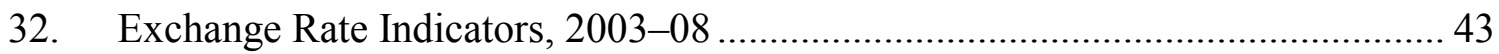




\section{TAX SUMMARY ${ }^{1}$}

\section{Taxation of Individuals}

\section{Direct TaXes}

Nature of Tax: Residents are taxed on their worldwide income. Nonresidents are taxed on income sourced from the Solomon Islands. The tax year is the calendar year.

Chargeable Income is income according to ordinary concepts and includes income from business, employment, lease and rent, dividends, interest, discounts, pensions, charges, annuities, and alimony received. Income from employment includes wages, salaries, other cash allowances, benefits-in-kind, and any private expenditure either paid for or reimbursed by an employer. Certain costs borne by an employer are excluded from employment income, such as employee home passage, medical services, and contributions paid to an approved pension fund. Many types of income are subject to presumptive or final taxes. These, with few specific exceptions, include income from employment, dividends, and interest. As such with effect from January 1, 2006, most employees or those individuals not in business are not required to lodge income tax returns.

\begin{tabular}{|cc|}
\hline Resident Individuals & Rates (in percent) \\
Up to $\$ 15,000$ & 11 \\
$\$ 15,001-\$ 30,000$ & 23 \\
$\$ 30,001-\$ 60,000$ & 35 \\
$\$ 60,001$ and up & 40 \\
\hline
\end{tabular}

Exemptions and deductions: Normal business expenses are deductible against chargeable income. A $\$ 7,800$ personal exemption applies to both resident and nonresident individuals. This general exemption is subject to apportionment on a pro-rata basis should the individual commence work part way through the year. In addition, the first $\$ 5,000$ of interest income on deposits made with any savings bank or on fixed deposit accounts with any bank in the Solomon Islands are excluded from chargeable income. Alimony payments are also deductible against chargeable income. Residents are able to claim foreign tax credits for any overseas income tax.

\footnotetext{
${ }^{1}$ As of June 2008.
} 


\section{Taxation of Companies}

Nature of tax: Tax is payable on the worldwide income of a resident company, whereas a nonresident company is taxed on income accrued in or derived from the Solomon Islands. The tax year is the calendar year, although any person may apply for a substituted accounting period.

Companies incorporated in the Solomon Islands are residents. Resident companies are taxed at a flat rate of 30 percent, with nonresident companies being subject to a 35 percent tax rate. To prevent the double taxation of corporate profits, a deduction is allowed for any dividend paid where dividend withholding tax has been correctly deducted and remitted. The rate of dividend withholding tax for a resident company or individual shareholder is 20 percent with 30 percent applying to a nonresident or a resident body of persons other than a corporation. The withholding tax deducted is final for a nonresident, a resident body of persons other than a company, and a resident individual where the total amount of dividends, directors fees, and interest income for the year is less than $\$ 10,000.00$.

A provisional payment system for income tax applies to companies and businesses. Quarterly payments of taxes are made in the current year, based on the assessed income of the previous year. Such payments are credited against the tax for the year when the annual assessment is made. For those taxpayers in a loss situation, a turnover tax of 0.5 percent of gross income applies. The maximum of income tax payable for these taxpayers is $\$ 20,000.00$.

Exemptions and deductions: Both resident and nonresident investors undertaking commercial, economic, industrial or professional activity in the Solomon Islands may apply to the Commissioner of Inland Revenue for an exemption from income tax. Depending upon the type of enterprise, the tax holiday may be for periods ranging from three to ten years. Strict criteria apply, with the benefits and costs to the national interest of the country being the chief consideration. Where the original investor proposes additional investment to the value of $\$ 10,000,000.00$ or more, and has satisfied the conditions stipulated in relation to the original investment, the Commissioner may grant a further tax holiday of up to five years. Rural community developments with investment of $\$ 5,000,000$ or more may also qualify for tax holiday. The Commissioner may also grant full or partial exemptions from income tax for certain activities:

- Tourist hotels, resorts and other tourist oriented projects (5 years);

- Export projects (3 to 6 years);

- Agricultural, forestry, animal husbandry and fisheries activities (profits for 5 of 10 years);

- Factory construction (40 percent depreciation in year one, then 5 percent per year thereafter); 
- 200 percent deduction for costs of higher education courses (local and overseas) and apprentices at the Solomon Islands College of Higher Education; and

- 150 percent deduction for inter-provincial transport costs of raw material and qualifying products.

The Minister of Finance also has the discretion to exempt any income or class of income by way of Order.

Statutory exemption from income tax applies to a range of government, quasi government, nonprofit, religious and sporting bodies.

Losses are able to be carried forward for five years and be set off against future profits if majority shareholding remains unchanged. Bond issues are exempt until the amount capitalized is distributed. Business license fees paid to provinces may be claimed as a deduction or treated as prepayments of income tax. Accelerated depreciation is provided for capital expenditure in timber and mining operations, while certain expenditures on agricultural development, including for coconuts, palm oil, and cocoa, can be written off in full in the year they are incurred.

\section{Social Security Contributions}

Nature of tax: Compulsory social security scheme for all public and private employees. A total of 7.5 percent of earnings is paid by the employer, and 5 percent by the employee.

\section{Capital Gains Tax}

None, except for business assets.

\section{Withholding Taxes}

Certain payments made to both resident and nonresident taxpayers are subject to withholding taxes. The list below specifies the nature of the payment subject to withholding tax. The payer is required to withhold the specified percentage of gross payment and forward such to the Commissioner on behalf of the payee. Depending on the circumstances of the taxpayer, the withholding tax may be deemed final or interim. 


\begin{tabular}{|l|c|}
\hline & Rates (in percent) \\
\hline Companies incorporated in the Solomon Islands & 30 \\
Other companies & 35 \\
Residents: & \\
Income from contracting & 7.5 \\
Royalties & 10 \\
Fishing operations & 10 \\
Lease of property & 10 \\
Sale of marine products & 10 \\
Stevedoring services & 15 \\
Nonresidents: & \\
Interest & 15 \\
Professional Services & 20 \\
Royalties & 15 \\
Income from contracting & 7.5 \\
Income from ships and aircraft & 5 \\
Insurance premiums & 15 \\
Film rental & 5 \\
Remuneration to pole and line or long-line fishermen & 10 \\
Remuneration to Purse Seiner fishermen & 15 \\
Lease income & 15 \\
Interest paid overseas by a mining company & As provided by the Minister \\
Management services & 35 \\
\hline
\end{tabular}

\begin{tabular}{|l|c|}
\hline \multicolumn{1}{|c|}{ Dividend Taxes } & Rates (in percent) \\
\hline Residents & 20 \\
Nonresidents & 30 \\
\hline
\end{tabular}

\section{Excise Duties}

\section{INDIRECT TAXES}

Nature of tax: An excise duty is levied on plugs, twist, fig, stick, cake, and coarse cut tobacco, and on beer and cigarettes produced in Solomon Islands. Imported excisable goods pay import duties at the following specific rates:

\begin{tabular}{|c|c|}
\hline & Rates \\
\hline Beer & $\$ 4.80$ per liter \\
\hline Tobacco & $\$ 48.00$ per $\mathrm{kg}$. \\
\hline Cigarettes & $\begin{array}{l}\$ 0.185 \text { per stick or } \$ 277.00 \text { per kg for } \\
\text { cigarettes containing tobacco with a tar } \\
\text { content more than } 16 \text { milligrams per } \\
\text { cigarette. }\end{array}$ \\
\hline
\end{tabular}




\section{Goods Tax}

Nature of tax: Imposed and levied on the final wholesale price of goods that are produced domestically or imported, whether new or used. Manufacturers and wholesale merchants are required to register with the Inland Revenue and are obliged to calculate the tax payable, charge and collect the tax from customers, and pay the tax to the Inland Revenue Division each month. Nonregistered importers, or those that choose not to quote their registration on import documentation, pay Goods Tax before goods are cleared by Customs. The value for Goods Tax in these cases is 130 per cent of the sum of the Cost-in-Freight Customs value and the Customs duty.

\begin{tabular}{|l|c|}
\hline & Rates \\
\hline Imported goods & 15 \\
Locally manufactured goods & 10 \\
\hline
\end{tabular}

Exemptions and deductions: Exemption from Goods Tax applies to the following goods:

- $\quad$ Drugs and medicine, and surgical tools;

- $\quad$ Educational, scientific and religious goods, works of art and antiques;

- $\quad$ Goods for use by governments, representatives of governments, and public bodies;

- $\quad$ Goods manufactured and sold by small businesses;

- Containers;

- $\quad$ Rice; and

- Miscellaneous: contraceptives, goods designed for use by disabled people, goods purchased for export, coffins, bird food, aquatic and marine food, coins, goods used as aids to manufacture, safety equipment, aircraft, buses, flags, awards, fire equipment.

The Minister of Finance has by way of Order discretion to exempt specific goods from goods tax. Official guidelines have been released setting out the criteria for exemption applications. The guidelines approved by the Minister of Finance in June 2006 state that exemptions will be assessed on the benefits and costs to the national interest and requires that details of all exemptions granted be Gazetted. Both the Inland Revenue Division and Customs Division have established Exemption Committees to consider applications and provide advice to the Minister.

\section{Import Taxes}

Nature of tax: Levied at an ad valorem rate on the c.i.f. value of imports, although specific rates apply on alcoholic beverages, tobacco products, and fuel. 


\begin{tabular}{|l|c|}
\hline & $\begin{array}{c}\text { Rates (in percent, unless } \\
\text { otherwise noted) }\end{array}$ \\
\hline Foodstuff & $0-10$ \\
Minerals, fuels & $0-10$, plus specific rates \\
Chemicals & $5-10$ \\
Machinery and transport equipment & $5-10$ \\
Domestic products & $0-10$ \\
Beer & $\$ 10$ per liter \\
Cigarettes & $\$ 0.42$ per stick \\
Tobacco and cigars & $\$ 500.00$ per kg \\
\hline
\end{tabular}

Exemptions and deductions: Based on the national interest exemption from import duties may be considered for imports of capital inputs for: (i) new businesses commencing in the Solomon Islands; (ii) modernization or expansion of existing business activities; and (iii) rural community development. The following imports are also exempt:

- $\quad$ Goods for all levels of government but excluding certain consumable goods;

- Goods imported under diplomatic and similar privileges, or under memoranda of understanding or agreements with foreign governments and international organizations or between the Solomon Islands Government and private companies;

- $\quad$ Aircraft parts and equipment, aircraft fuel, and lubricants for use in foreign aircraft;

- Containers, coverings, packages, etc;

- Cultural and educational articles; health goods for relief work and charity; fire fighting equipment; goods for volunteer organizations; marine and port equipment; fuel and life-saving equipment; religious goods; scientific and meteorological equipment; goods for telecommunications systems;

- Commercial fishing gear and equipment;

- Most capital goods and agricultural equipment;

- $\quad$ Raw materials and industrial imports to manufacturing;

- Goods imported for Rural Community Development Projects that are fully funded from Rural Community Development funds;

- Goods for tourism or imported under investment incentives;

- Goods imported by armed forces, demolition explosives and machinery, clothes and accessories required for civil servants;

- Medals and decorations; uniforms and insignias of youth organizations; advertising samples; portraits, films, recordings for personal use.

Partial exemptions (where the duty rate is reduced to 2 per cent) applies to goods for direct use in agriculture, horticulture, fishery, forestry and mining, and to certain inputs to particular processing operations. In addition to the above, applications for ad hoc exemptions from import or export duty are considered by a committee that makes recommendations to the Minister for Finance for decision. 


\section{Sales Tax}

Nature of tax: A sales tax is levied at the retail level for specified services.

\begin{tabular}{|l|c|}
\hline Local and overseas telecommunications & $\begin{array}{c}\text { Rates (in percent, unless } \\
\text { otherwise noted) }\end{array}$ \\
Restaurant services & 10 \\
Tickets for overseas travel & 10 \\
Tickets for domestic air travel & $\$ 10$ \\
Tickets for domestic sea travel & $\$ 10$ \\
Hire of video & 10 \\
Movie tickets & $\$ 2$ per tape \\
Accounting, legal, and security services & $\$ 0.50$ per ticket \\
Motor vehicle rental & 10 \\
Real estate agency services & 10 \\
Professional services & 10 \\
Petroleum and diesel & 10 \\
Video equipment hires & $\$ 0.1$ per liter \\
Computer equipment hires & 10 \\
Hire of vessel and aircraft & 10 \\
Hire of plant machinery and equipment & 10 \\
Electronic repairs and equipment & 10 \\
Hairdresser and barber shops including beauty parlors & 10 \\
Casinos & 10 \\
Laundry services & $\$ 5$ per entrance \\
Tire services & 10 \\
\hline
\end{tabular}

\section{Export Taxes}

Nature of tax: Levied only on various land and marine fauna shells, gold, metal scrap, logs and lumber, fish, and various agricultural products. The valuation base is the f.o.b. price or value of the export, except in the case of timber and some fish where the value for duty is a "determined value" adjustable from time to time.

Exemptions and deductions: Spices, coconut, palm oil, canned fish, rice, some shells, copra, biscuits, beer, manufactured tobacco. Exemptions for export taxes on logs are granted on a case-by-case basis. Recently, the Minister of Finance announced the revocation of some logging duty exemptions. 


\begin{tabular}{|l|c|}
\hline & $\begin{array}{c}\text { Rates (in percent, unless otherwise } \\
\text { noted) }\end{array}$ \\
\hline Trocus, green snail and black lip shells & 30 \\
Gold & 5 \\
Metal scrap — nonferrous & $\$ 1.5 /$ metric ton \\
Metal scrap & 15 \\
Timber logs: & \\
$\quad$ If valued up to $\$ 500 / \mathrm{m}^{3}$ & 25 percent (of the determined value) \\
$\ldots$..If valued between $\$ 550-850 / \mathrm{m} 3$ & $\$ 137.50$ plus 40 percent of difference \\
If valued more than $\$ 850 / \mathrm{m} 3$ & $\$ 257.50$ plus 60 percent of difference \\
Fresh, chilled, or frozen fish (some) & 5 \\
Dried, salted, smoked or cooked fish & 20 \\
Various other agricultural, forest, marine products & \\
\hline
\end{tabular}

\section{OTher TAXES AND ChARgeS}

\section{Business Licenses}

Nature of tax: Annual levy payable by retail traders at various rates.

\section{Other Licenses}

Nature of tax: Licenses on motor vehicles, driving permits, fishing vessels, work permits, and firearms.

\begin{tabular}{|l|l|}
\hline Motor vehicles & $\begin{array}{l}\text { Specific rates according to engine displacement } \\
\text { and weight varying from } \$ 150 \text { to } \$ 2,500\end{array}$ \\
Driving license & $\$ 75$ \\
Dealer license & $\$ 10,000$ \\
Work permits application & $\$ 200$, and upon approval $\$ 500$ for two years \\
\hline
\end{tabular}

\section{Stamp Duties}

Nature of tax: Levied on agreements, insurance policies, bills of sales and exchange, promissory notes, and various property and title transfers. Some are ad valorem, some specific. The duty varies from $\$ 50$ on any instrument, and between $2.0-4.0$ percent depending on the value of property.

Exemptions and deductions: Bills of exchange by any bank for government purposes. 
Table 1. Solomon Islands: Summary of Real Sector Indicators, 2001-07

\begin{tabular}{|c|c|c|c|c|c|c|c|}
\hline & 2001 & 2002 & 2003 & 2004 & 2005 & 2006 & $\begin{array}{r}\text { Est. } \\
2007\end{array}$ \\
\hline \multicolumn{8}{|l|}{ Real GDP 1/ } \\
\hline \multirow[t]{2}{*}{ Percentage growth } & -8.0 & -2.8 & 6.5 & 8.0 & 5.0 & 6.1 & 10.3 \\
\hline & \multicolumn{7}{|c|}{ (Contribution to growth; in percent) } \\
\hline Primary & -1.2 & 0.9 & 4.9 & 3.7 & 0.6 & 2.2 & 2.2 \\
\hline Agriculture & -0.5 & 0.3 & 1.1 & 0.1 & 0.5 & 0.1 & 0.9 \\
\hline Forestry & 0.1 & 0.0 & 2.1 & 2.9 & 0.9 & 0.6 & 2.4 \\
\hline Fishing & -0.8 & 0.6 & 1.7 & 0.7 & -0.7 & 1.5 & -1.1 \\
\hline Secondary 2/ & -0.7 & -0.1 & -0.3 & 0.2 & 0.5 & 0.4 & 0.6 \\
\hline Tertiary $3 /$ & -2.2 & -2.5 & -1.5 & 1.5 & 1.2 & 2.1 & 3.1 \\
\hline Nonmonetary sector & 0.7 & 0.8 & 0.8 & 1.0 & 0.8 & 0.9 & 1.1 \\
\hline \multirow[t]{2}{*}{ Indirect taxes and subsidies } & -4.6 & -1.8 & 2.7 & 1.7 & 1.8 & 0.6 & 3.3 \\
\hline & \multicolumn{7}{|c|}{ (In 1,000 metric tons, unless otherwise specified) } \\
\hline \multicolumn{8}{|l|}{ Production of major commodities } \\
\hline Logging (1,000 cubic meters) & 534.0 & 550.0 & 736.0 & 1,043 & 1,118 & 1,130 & 1,446 \\
\hline Fish & 17.7 & 18.5 & 28.0 & 27.2 & 23.8 & 29.6 & 21.1 \\
\hline Copra & 1.7 & 1.7 & 14.8 & 21.8 & 26.2 & 21.2 & 27.9 \\
\hline Cocoa & 2.0 & 2.9 & 4.6 & 4.2 & 4.9 & 3.8 & 4.5 \\
\hline \multirow[t]{2}{*}{ Palm oil } & $\ldots$ & $\ldots$ & $\ldots$ & $\ldots$ & $\ldots$ & 5.4 & 17.2 \\
\hline & \multicolumn{7}{|c|}{ (Annual percentage change) } \\
\hline Consumer prices (average) & 7.6 & 9.3 & 10.0 & 6.9 & 7.4 & 11.2 & 7.7 \\
\hline Consumer prices (end-of-period) & 6.5 & 15.3 & 3.7 & 7.6 & 8.6 & 9.9 & 10.9 \\
\hline
\end{tabular}

Sources: Data provided by the Solomon Islands authorities; and Fund staff estimates.

1/ Estimates at 1992 factor cost.

2/ Includes mining and exploration, manufacturing, electricity and water, and construction.

$3 /$ Includes retail and wholesale trade, transportation and communications, finance, and other services. 
Table 2. Solomon Islands: Real GDP Growth Rates by Sectoral Origin, 2004-07

(Annual percentage change)

\begin{tabular}{|c|c|c|c|c|}
\hline & 2004 & 2005 & 2006 & $\begin{array}{r}\text { Est. } \\
2007\end{array}$ \\
\hline Primary sector & 20.4 & 3.2 & 11.1 & 10.3 \\
\hline Agriculture & 3.4 & 11.2 & 2.3 & 21.2 \\
\hline Forestry & 40.0 & 9.4 & 6.3 & 24.5 \\
\hline Fishing & 10.6 & -9.6 & 25.1 & -15.2 \\
\hline Secondary sector & 3.5 & 9.3 & 7.7 & 12.2 \\
\hline Mining and exploration & 3.1 & 0.0 & 0.0 & 0.0 \\
\hline Manufacturing & 1.1 & 1.0 & 2.8 & 4.7 \\
\hline Electricity and water & 0.7 & 17.5 & 1.8 & 12.0 \\
\hline Construction & 27.0 & 16.0 & 33.5 & 34.0 \\
\hline Tertiary & 4.1 & 3.5 & 6.2 & 9.2 \\
\hline Retail and wholesale trade & 3.1 & 1.7 & 0.0 & 6.5 \\
\hline Transportation and communications & 3.4 & 1.9 & 27.9 & 20.4 \\
\hline Finance & 2.6 & 1.0 & 1.9 & 9.2 \\
\hline Other services & 6.1 & 7.1 & 6.5 & 6.7 \\
\hline Monetary GDP at factor cost & 9.6 & 5.5 & 7.4 & 12.5 \\
\hline Nonmonetary GDP at factor cost & 2.8 & 2.8 & 2.8 & 2.8 \\
\hline Food & 2.8 & 2.8 & 2.8 & 2.8 \\
\hline Construction & 2.8 & 2.8 & 2.8 & 2.8 \\
\hline Real GDP at market prices & 9.2 & 3.9 & 8.0 & 9.8 \\
\hline \multicolumn{5}{|l|}{ Memorandum item: } \\
\hline $\begin{array}{l}\text { Nominal GDP at current market prices } \\
\text { (in millions of SI dollars) }\end{array}$ & $1,961.1$ & $2,207.5$ & $2,542.3$ & $2,971.3$ \\
\hline
\end{tabular}

Sources: Data provided by the Solomon Islands authorities; and Fund staff estimates. 
Table 3. Solomon Islands: Honiara Retail Price Index, 2004-08 1/

\begin{tabular}{|c|c|c|c|c|c|c|c|c|c|c|c|}
\hline Weight & $\begin{array}{l}\text { Food } \\
429\end{array}$ & $\begin{array}{r}\text { Drink } \\
\text { and } \\
\text { Tobacco } \\
47\end{array}$ & $\begin{array}{r}\text { Clothing } \\
\text { and } \\
\text { Footwear } \\
38\end{array}$ & $\begin{array}{r}\text { Housing } \\
\text { and } \\
\text { Utilites } \\
181\end{array}$ & $\begin{array}{r}\text { Household } \\
\text { Operations } \\
47\end{array}$ & 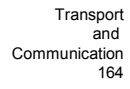 & $\begin{array}{r}\text { Recreational } \\
\text { Health and } \\
\text { Other Services } \\
76\end{array}$ & $\begin{array}{r}\text { Miscellaneous } \\
\text { Items } \\
18\end{array}$ & $\begin{array}{r}\text { Imported } \\
\text { Items } \\
602\end{array}$ & $\begin{array}{r}\text { Local } \\
\text { Items } \\
429\end{array}$ & $\begin{array}{l}\text { All } \\
\text { Items } \\
1,000\end{array}$ \\
\hline 2004 & 92.3 & 100.0 & 95.5 & 83.8 & 92.0 & 76.4 & 86.2 & 93.0 & 92.9 & 313.0 & 89.1 \\
\hline 2005 & 98.6 & 98.2 & 96.7 & 95.0 & $\begin{array}{l}92.0 \\
96.9\end{array}$ & $\begin{array}{l}10.4 \\
90.9\end{array}$ & $\begin{array}{l}80.2 \\
96.4\end{array}$ & $\begin{array}{l}97.3 \\
97.3\end{array}$ & $\begin{array}{l}97.9 \\
97.8\end{array}$ & 97.2 & $\begin{array}{l}89.1 \\
96.7\end{array}$ \\
\hline 2006 & 108.3 & 109.4 & 115.0 & 107.0 & 107.8 & 105.7 & 112.5 & 109.1 & 108.4 & 108.2 & $\begin{array}{l}108.2 \\
\end{array}$ \\
\hline 2007 & 114.8 & 113.8 & 119.3 & 127.4 & 117.4 & 110.0 & 113.7 & 120.5 & 116.7 & 116.5 & 116.6 \\
\hline \multicolumn{12}{|l|}{2004} \\
\hline January & 88.3 & 103.8 & 95.2 & 81.2 & 90.1 & 72.2 & 83.7 & 91.4 & 89.8 & 302.6 & 86.2 \\
\hline February & 90.8 & 105.0 & 95.3 & 81.9 & 90.9 & 74.0 & 84.0 & 92.1 & 91.6 & 308.2 & 87.8 \\
\hline March & 90.6 & 105.1 & $\begin{array}{l}95.3 \\
05.3\end{array}$ & 81.7 & 91.5 & $\begin{array}{l}74.6 \\
75.6\end{array}$ & 84.6 & 91.7 & 92.4 & $\begin{array}{l}307.0 \\
311.8\end{array}$ & 87.9 \\
\hline $\begin{array}{l}\text { April } \\
\text { May }\end{array}$ & $\begin{array}{l}92.8 \\
93.0\end{array}$ & $\begin{array}{l}99.6 \\
99.6\end{array}$ & $\begin{array}{l}95.3 \\
956\end{array}$ & $\begin{array}{l}80.8 \\
81.0\end{array}$ & $\begin{array}{l}92.1 \\
92.2\end{array}$ & $\begin{array}{l}75.0 \\
75.3\end{array}$ & $\begin{array}{l}88.6 \\
87.3\end{array}$ & $\begin{array}{l}92.5 \\
92.5\end{array}$ & $\begin{array}{l}92.1 \\
92.1\end{array}$ & $\begin{array}{l}311.8 \\
3140\end{array}$ & $\begin{array}{l}88.6 \\
89.0\end{array}$ \\
\hline $\begin{array}{l}\text { May } \\
\text { June }\end{array}$ & $\begin{array}{l}93.0 \\
92.8\end{array}$ & $\begin{array}{l}99.6 \\
100.0\end{array}$ & $\begin{array}{l}95.6 \\
95.6\end{array}$ & $\begin{array}{l}81.0 \\
81.0\end{array}$ & $\begin{array}{l}92.2 \\
92.2\end{array}$ & $\begin{array}{l}75.3 \\
75.3\end{array}$ & $\begin{array}{l}87.3 \\
87.3\end{array}$ & $\begin{array}{l}92.5 \\
92.9\end{array}$ & $\begin{array}{l}92.1 \\
92.7\end{array}$ & $\begin{array}{l}314.0 \\
312.5\end{array}$ & $\begin{array}{l}89.0 \\
89.0\end{array}$ \\
\hline July & $\begin{array}{l}92.0 \\
93.3\end{array}$ & 100.1 & $\begin{array}{l}95.6 \\
95.6\end{array}$ & 83.1 & 92.2 & 77.3 & 87.2 & 93.4 & $\begin{array}{l}92.1 \\
93.7\end{array}$ & 314.7 & 89.7 \\
\hline August & 92.6 & 97.6 & 95.6 & 82.4 & 92.4 & 77.1 & 87.1 & 93.6 & 93.2 & 312.8 & 89.2 \\
\hline September & 93.8 & 102.0 & 95.6 & 85.8 & 92.4 & 78.1 & 87.1 & 93.8 & 94.6 & 317.6 & 90.5 \\
\hline $\begin{array}{l}\text { October } \\
\text { Novemer }\end{array}$ & $\begin{array}{l}93.4 \\
934\end{array}$ & $\begin{array}{l}95.5 \\
95.5 \\
95\end{array}$ & $\begin{array}{l}95.6 \\
956\end{array}$ & $\begin{array}{l}87.9 \\
886\end{array}$ & $\begin{array}{l}92.6 \\
92.7\end{array}$ & $\begin{array}{l}79.2 \\
79.1\end{array}$ & $\begin{array}{l}87.1 \\
87.2\end{array}$ & $\begin{array}{l}93.4 \\
94.0\end{array}$ & $\begin{array}{l}94.2 \\
94.2\end{array}$ & $\begin{array}{l}317.9 \\
318 .\end{array}$ & $\begin{array}{l}90.4 \\
90.5\end{array}$ \\
\hline $\begin{array}{l}\text { Noverember } \\
\text { December }\end{array}$ & $\begin{array}{l}93.4 \\
93.1\end{array}$ & $\begin{array}{l}95.5 \\
96.7\end{array}$ & $\begin{array}{l}95.6 \\
95.5\end{array}$ & $\begin{array}{l}88.6 \\
90.0\end{array}$ & $\begin{array}{l}92.1 \\
92.9\end{array}$ & $\begin{array}{l}79.1 \\
79.6\end{array}$ & $\begin{array}{l}88.2 \\
87.2\end{array}$ & $\begin{array}{l}94.0 \\
94.1\end{array}$ & $\begin{array}{l}94.2 \\
94.2\end{array}$ & $\begin{array}{l}318.3 \\
318.8\end{array}$ & $\begin{array}{l}90.5 \\
90.7\end{array}$ \\
\hline \multicolumn{12}{|l|}{2005} \\
\hline January & $\begin{array}{l}93.9 \\
957\end{array}$ & 99.4 & 95.5 & 87.8 & 92.9 & 78.7 & 93.8 & 94.4 & 94.3 & 91.9 & 91.4 \\
\hline $\begin{array}{l}\text { February } \\
\text { March }\end{array}$ & $\begin{array}{l}95.7 \\
952\end{array}$ & $\begin{array}{l}99.3 \\
993\end{array}$ & $\begin{array}{l}95.5 \\
95.5\end{array}$ & $\begin{array}{l}88.2 \\
88.2\end{array}$ & 93.2 & $\begin{array}{l}79.1 \\
80.8\end{array}$ & $\begin{array}{l}93.7 \\
99.1\end{array}$ & 95.3 & 93.7 & $\begin{array}{l}92.8 \\
93.9\end{array}$ & 92.4 \\
\hline $\begin{array}{l}\text { March } \\
\text { April }\end{array}$ & $\begin{array}{l}95.2 \\
98.5\end{array}$ & $\begin{array}{l}99.3 \\
943\end{array}$ & $\begin{array}{l}95.5 \\
956\end{array}$ & $\begin{array}{l}88.2 \\
92.4\end{array}$ & $\begin{array}{l}93.2 \\
96.5\end{array}$ & $\begin{array}{l}80.8 \\
80.3\end{array}$ & $\begin{array}{l}94.1 \\
95.7\end{array}$ & $\begin{array}{l}96.6 \\
96.2\end{array}$ & $\begin{array}{l}94.6 \\
95.2\end{array}$ & $\begin{array}{l}93.9 \\
93.5\end{array}$ & $\begin{array}{l}92.4 \\
94.6\end{array}$ \\
\hline $\begin{array}{l}\text { Aprin } \\
\text { May }\end{array}$ & $\begin{array}{l}98.5 \\
97.5\end{array}$ & $\begin{array}{l}94.3 \\
96.1\end{array}$ & $\begin{array}{l}95.6 \\
95.6\end{array}$ & $\begin{array}{l}92.4 \\
96.4\end{array}$ & $\begin{array}{l}96.5 \\
96.8\end{array}$ & $\begin{array}{l}80.3 \\
80.3\end{array}$ & 95.7 & $\begin{array}{l}96.2 \\
96.3\end{array}$ & $\begin{array}{l}98.2 \\
98.3\end{array}$ & $\begin{array}{l}93.5 \\
95.4\end{array}$ & $\begin{array}{l}94.6 \\
94.8\end{array}$ \\
\hline June & 99.5 & 96.1 & 95.6 & 96.0 & 97.0 & 97.7 & 95.8 & 96.9 & 98.8 & 95.4 & 97.9 \\
\hline July & 102.7 & 97.8 & 95.6 & 96.6 & 97.7 & 97.6 & 95.8 & 97.1 & 98.9 & 100.1 & 99.6 \\
\hline August & $\begin{array}{l}100.5 \\
097\end{array}$ & $\begin{array}{l}97.8 \\
077\end{array}$ & $\begin{array}{l}95.6 \\
0.56\end{array}$ & 97.0 & 97.9 & 98.0 & $\begin{array}{l}95.8 \\
90.8\end{array}$ & $\begin{array}{l}97.4 \\
97.6\end{array}$ & $\begin{array}{r}99.5 \\
09.8\end{array}$ & 102.5 & 98.7 \\
\hline $\begin{array}{l}\text { September } \\
\text { October }\end{array}$ & $\begin{array}{l}99.7 \\
997\end{array}$ & $\begin{array}{l}97.7 \\
996\end{array}$ & $\begin{array}{r}95.6 \\
1000\end{array}$ & $\begin{array}{l}97.0 \\
99.8\end{array}$ & $\begin{array}{l}97.6 \\
99.9\end{array}$ & $\begin{array}{l}98.0 \\
99.0\end{array}$ & $\begin{array}{c}95.8 \\
10.0\end{array}$ & $\begin{array}{c}97.6 \\
100.0\end{array}$ & $\begin{array}{l}99.8 \\
99.5\end{array}$ & $\begin{array}{r}101.0 \\
99.7\end{array}$ & $\begin{array}{l}98.3 \\
99.6\end{array}$ \\
\hline $\begin{array}{l}\text { October } \\
\text { November }\end{array}$ & $\begin{array}{l}99.7 \\
99.8\end{array}$ & $\begin{array}{l}99.6 \\
99.6\end{array}$ & $\begin{array}{l}100.0 \\
100.0\end{array}$ & $\begin{array}{r}99.8 \\
100.2\end{array}$ & $\begin{array}{r}90.9 \\
100.0\end{array}$ & $\begin{array}{l}90.0 \\
101.4\end{array}$ & $\begin{array}{l}100.0 \\
100.0\end{array}$ & $\begin{array}{l}100.0 \\
100.0\end{array}$ & $\begin{array}{r}99.5 \\
100.6\end{array}$ & $\begin{array}{l}99.7 \\
99.8\end{array}$ & $\begin{array}{l}99.6 \\
100.1\end{array}$ \\
\hline December & $\begin{array}{l}93.0 \\
100.6\end{array}$ & 100.8 & 100.0 & 100.0 & 100.1 & 99.7 & 100.0 & 100.2 & 99.9 & 100.5 & $\begin{array}{l}100.2 \\
100.2\end{array}$ \\
\hline \multicolumn{12}{|l|}{2006} \\
\hline January & 102.5 & 105.3 & 113.9 & 105.4 & 106.9 & 103.8 & 113.0 & 107.2 & 105.0 & 104.8 & 104.9 \\
\hline February & 102.7 & 104.6 & 113.9 & 104.8 & 107.1 & 102.9 & 112.3 & 107.2 & 104.5 & 104.7 & 104.6 \\
\hline March & 104.5 & 102.2 & 113.7 & 105.0 & 107.3 & 103.3 & 112.3 & 107.2 & 105.1 & 105.6 & 105.4 \\
\hline April & $\begin{array}{l}107.0 \\
1096\end{array}$ & $\begin{array}{r}99.8 \\
1086\end{array}$ & $\begin{array}{l}113.8 \\
1139\end{array}$ & $\begin{array}{l}106.2 \\
1066\end{array}$ & 107.2 & $\begin{array}{l}105.1 \\
1050\end{array}$ & $\begin{array}{l}112.3 \\
1123\end{array}$ & $\begin{array}{l}107.2 \\
1070\end{array}$ & $\begin{array}{l}100.4 \\
106.6\end{array}$ & $\begin{array}{l}107.2 \\
109.7\end{array}$ & $\begin{array}{l}106.9 \\
108.5\end{array}$ \\
\hline $\begin{array}{l}\text { May } \\
\text { June }\end{array}$ & $\begin{array}{l}109.6 \\
110.6\end{array}$ & $\begin{array}{l}108.6 \\
108.5\end{array}$ & $\begin{array}{l}113.9 \\
113.9\end{array}$ & $\begin{array}{l}106.6 \\
106.9\end{array}$ & $\begin{array}{l}107.2 \\
107.3\end{array}$ & $\begin{array}{l}105.0 \\
105.9\end{array}$ & $\begin{array}{l}1122.3 \\
112.3\end{array}$ & $\begin{array}{l}107.0 \\
107.2\end{array}$ & $\begin{array}{l}106.6 \\
108.7\end{array}$ & $\begin{array}{l}109.7 \\
109.4\end{array}$ & $\begin{array}{l}108.5 \\
109.1\end{array}$ \\
\hline July & 111.8 & $\begin{array}{l}10.5 \\
113.1\end{array}$ & $\begin{array}{l}113.9 \\
\end{array}$ & $\begin{array}{l}107.2 \\
107.9\end{array}$ & 107.4 & $\begin{array}{l}100.9 \\
106.3\end{array}$ & $\begin{array}{l}112.3 \\
112.3\end{array}$ & 107.0 & $\begin{array}{l}109.5 \\
109.5\end{array}$ & $\begin{array}{l}110.4 \\
110.2\end{array}$ & $\begin{array}{l}109.9 \\
109.9\end{array}$ \\
\hline August & 111.3 & 113.3 & 114.4 & 107.3 & 108.2 & 106.7 & 112.3 & 108.0 & 109.7 & 110.0 & 109.9 \\
\hline September & 109.5 & 112.5 & 114.4 & 107.3 & 108.2 & 106.7 & 112.3 & 108.3 & 109.8 & 108.7 & 109.1 \\
\hline October & $\begin{array}{l}110.4 \\
1102\end{array}$ & $\begin{array}{l}117.3 \\
1152\end{array}$ & $\begin{array}{l}116.8 \\
1185\end{array}$ & 108.4 & 108.6 & 107.8 & 112.8 & $\begin{array}{l}111.6 \\
115.5\end{array}$ & 111.4 & 109.6 & $\begin{array}{l}110.3 \\
1104\end{array}$ \\
\hline $\begin{array}{l}\text { November } \\
\text { December }\end{array}$ & $\begin{array}{l}110.2 \\
109.9\end{array}$ & $\begin{array}{l}115.2 \\
111.8\end{array}$ & $\begin{array}{l}118.5 \\
118.9\end{array}$ & $\begin{array}{l}109.2 \\
109.5\end{array}$ & $\begin{array}{l}109.1 \\
109.2\end{array}$ & $\begin{array}{l}107.6 \\
107.6\end{array}$ & $\begin{array}{l}\begin{array}{l}1122.9 \\
112.9\end{array} \\
\end{array}$ & $\begin{array}{l}\begin{array}{l}1115.3 \\
116.2\end{array} \\
\end{array}$ & $\begin{array}{l}111.9 \\
112.1\end{array}$ & $\begin{array}{l}109.4 \\
1090\end{array}$ & $\begin{array}{l}110.4 \\
1102\end{array}$ \\
\hline \multicolumn{12}{|l|}{2007} \\
\hline January & 111.8 & 114.6 & 118.4 & 126.3 & 112.7 & 109.9 & 113.5 & 113.5 & 113.6 & 115.4 & 114.7 \\
\hline February & $\begin{array}{l}109.7 \\
110.1\end{array}$ & & 119.6 & $\begin{array}{l}126.7 \\
1167\end{array}$ & $\begin{array}{l}110.1 \\
111.3\end{array}$ & 108.0 & $\begin{array}{l}112.9 \\
113.5\end{array}$ & $\begin{array}{l}116.7 \\
1119\end{array}$ & 113.0 & 113.8 & $\begin{array}{l}113.5 \\
113.7\end{array}$ \\
\hline $\begin{array}{l}\text { March } \\
\text { Aprii }\end{array}$ & $\begin{array}{l}110.1 \\
1116\end{array}$ & $\begin{array}{l}114.5 \\
115.6\end{array}$ & $\begin{array}{l}119.6 \\
119.4\end{array}$ & $\begin{array}{l}126.7 \\
126.3\end{array}$ & $\begin{array}{l}1111.3 \\
1115\end{array}$ & $\begin{array}{l}108.0 \\
108\end{array}$ & 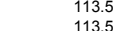 & $\begin{array}{l}114.9 \\
114.3\end{array}$ & $\begin{array}{l}113.1 \\
113.3\end{array}$ & $\begin{array}{l}114.2 \\
115.3\end{array}$ & $\begin{array}{l}113.7 \\
114.5\end{array}$ \\
\hline $\begin{array}{l}\text { April } \\
\text { May }\end{array}$ & $\begin{array}{l}171.6 \\
112.6\end{array}$ & $\begin{array}{l}715.6 \\
115.1\end{array}$ & $\begin{array}{l}179.4 \\
120.2\end{array}$ & $\begin{array}{l}126.3 \\
126.1\end{array}$ & 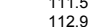 & $\begin{array}{l}108.8 \\
1090\end{array}$ & $\begin{array}{l}113.5 \\
113.5\end{array}$ & $\begin{array}{l}1114.3 \\
113.5\end{array}$ & $\begin{array}{l}713.3 \\
114.5\end{array}$ & $\begin{array}{l}175.3 \\
1153\end{array}$ & $\begin{array}{l}1144.5 \\
1150\end{array}$ \\
\hline June & 113.0 & 114.6 & $\begin{array}{l}120.2 \\
120.2\end{array}$ & 126.3 & 113.0 & 109.9 & 113.5 & 113.5 & $\begin{array}{l}115.1 \\
\text {. }\end{array}$ & 115.4 & 115.3 \\
\hline July & 113.2 & 108.2 & 120.2 & 126.9 & 113.2 & 109.0 & 113.5 & 113.5 & 114.9 & 115.1 & $\begin{array}{l}115.0 \\
1153\end{array}$ \\
\hline August & 113.4 & 109.4 & 117.0 & 127.7 & 113.2 & 109.2 & $\begin{array}{r}113.5 \\
11.4\end{array}$ & 113.4 & 115.2 & 115.3 & $\begin{array}{l}115.3 \\
1177\end{array}$ \\
\hline September & $\begin{array}{l}116.4 \\
1183\end{array}$ & $\begin{array}{l}107.1 \\
111.9\end{array}$ & $\begin{array}{l}119.7 \\
1197\end{array}$ & $\begin{array}{l}128.0 \\
128 .\end{array}$ & $\begin{array}{l}126.3 \\
127 .\end{array}$ & 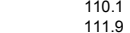 & $\begin{array}{l}1144.4 \\
114.4\end{array}$ & 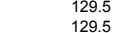 & $\begin{array}{l}119.1 \\
120.4\end{array}$ & $\begin{array}{l}116.8 \\
118.3\end{array}$ & $\begin{array}{l}117.7 \\
119.1\end{array}$ \\
\hline $\begin{array}{l}\text { October } \\
\text { Novemer }\end{array}$ & $\begin{array}{l}118.3 \\
1256\end{array}$ & $\begin{array}{l}1111.9 \\
111.9\end{array}$ & $\begin{array}{l}119.7 \\
1190\end{array}$ & $\begin{array}{l}128.2 \\
1298\end{array}$ & $\begin{array}{l}12,7.2 \\
128.9\end{array}$ & $\begin{array}{l}111.9 \\
1122\end{array}$ & $\begin{array}{l}1144.4 \\
1144\end{array}$ & $\begin{array}{l}129.5 \\
1368\end{array}$ & $\begin{array}{l}120.4 \\
1225\end{array}$ & $\begin{array}{l}118.3 \\
1229\end{array}$ & $\begin{array}{l}199.1 \\
1228\end{array}$ \\
\hline $\begin{array}{l}\text { Noverember } \\
\text { December }\end{array}$ & $\begin{array}{l}125.6 \\
121.6\end{array}$ & $\begin{array}{l}7111.9 \\
128.3\end{array}$ & $\begin{array}{l}119.0 \\
118.8\end{array}$ & $\begin{array}{l}129.8 \\
130.3\end{array}$ & $\begin{array}{l}128.9 \\
129.0\end{array}$ & $\begin{array}{l}\text { T112.2. } \\
114.1\end{array}$ & $\begin{array}{l}1114.4 \\
114.4\end{array}$ & $\begin{array}{l}1366.8 \\
136.8\end{array}$ & $\begin{array}{l}122.5 \\
126.1\end{array}$ & $\begin{array}{l}\begin{array}{l}222.9 \\
119.7\end{array}\end{array}$ & $\begin{array}{l}122.8 \\
122.2\end{array}$ \\
\hline \multicolumn{12}{|l|}{2008} \\
\hline January & 123.1 & 126.8 & 118.8 & 138.6 & 128.7 & 116.1 & 117.3 & 136.2 & 127.9 & 122.7 & 124.8 \\
\hline February & 124.9 & 126.8 & 118.6 & 138.6 & 128.9 & 115.8 & 117.3 & 137.6 & 127.8 & 124.1 & 125.6 \\
\hline March & 123.1 & 122.7 & 118.6 & 138.7 & 129.1 & 116.7 & 117.3 & 137.9 & 128.1 & 122.7 & 124.8 \\
\hline April & 129.7 & $\begin{array}{l}125.1 \\
1235\end{array}$ & $\begin{array}{l}119.2 \\
190 .\end{array}$ & $\begin{array}{l}142.0 \\
142.3\end{array}$ & $\begin{array}{l}129.9 \\
1319\end{array}$ & $\begin{array}{l}119.5 \\
121 .\end{array}$ & $\begin{array}{l}117.3 \\
117.3\end{array}$ & 137.0 & 133.5 & 125.8 & 128.8 \\
\hline $\begin{array}{l}\text { May } \\
\text { June }\end{array}$ & $\begin{array}{l}141.8 \\
147.1\end{array}$ & $\begin{array}{l}123.5 \\
122.9\end{array}$ & $\begin{array}{l}120.1 \\
120.5\end{array}$ & $\begin{array}{l}142.3 \\
1428\end{array}$ & $\begin{array}{l}131.9 \\
135.5\end{array}$ & $\begin{array}{l}121.3 \\
126.7\end{array}$ & $\begin{array}{l}1177.3 \\
1173\end{array}$ & $\begin{array}{l}141.0 \\
141.5\end{array}$ & $\begin{array}{l}142.8 \\
149.6\end{array}$ & $\begin{array}{l}129.1 \\
130.3\end{array}$ & $\begin{array}{l}134.5 \\
137.9\end{array}$ \\
\hline
\end{tabular}


Table 4. Solomon Islands: Formal Employment, 2004-07

\begin{tabular}{|c|c|c|c|c|}
\hline & $\begin{array}{r}\text { Est. } \\
2004\end{array}$ & $\begin{array}{l}\text { Est. } \\
2005\end{array}$ & $\begin{array}{r}\text { Est. } \\
2006\end{array}$ & 2007 \\
\hline Total & 52,121 & 56,559 & 59,161 & $\ldots$ \\
\hline \multicolumn{5}{|l|}{ Classification by industry } \\
\hline Agriculture & 6,342 & $\ldots$ & $\ldots$ & $\ldots$ \\
\hline Forestry & 3,482 & $\ldots$ & $\ldots$ & $\ldots$ \\
\hline Fishing & 5,114 & $\ldots$ & $\ldots$ & $\ldots$ \\
\hline Manufacturing $1 /$ & 1,476 & $\ldots$ & $\ldots$ & $\ldots$ \\
\hline Electricity and water & 469 & $\ldots$ & $\ldots$ & $\ldots$ \\
\hline Construction & 1,397 & $\ldots$ & $\ldots$ & $\ldots$ \\
\hline Trading 2/ & 3,274 & $\ldots$ & $\ldots$ & $\ldots$ \\
\hline Transportation and communications & 1,246 & $\ldots$ & $\ldots$ & $\ldots$ \\
\hline Finance & 806 & $\ldots$ & $\ldots$ & $\ldots$ \\
\hline Administration & 6,758 & $\ldots$ & $\ldots$ & $\ldots$ \\
\hline Other services & 21,757 & $\ldots$ & $\ldots$ & $\ldots$ \\
\hline
\end{tabular}

Sources: National Statistical Office; and Central Bank of Solomon Islands.

1/ Manufacturing includes mining.

2/ Trading includes retail, wholesale, restaurants, and hotels. 
Table 5. Solomon Islands: Central Government Operations, 2002-07

\begin{tabular}{|c|c|c|c|c|c|c|}
\hline & 2002 & 2003 & 2004 & 2005 & 2006 & $\begin{array}{r}\text { Est. } \\
2007\end{array}$ \\
\hline Total revenue and grants & 19.0 & 39.2 & 48.1 & 66.2 & 64.7 & 69.0 \\
\hline Total revenue & 16.3 & 21.2 & 26.3 & 29.7 & 32.3 & 36.8 \\
\hline Tax revenue & 15.6 & 19.4 & 23.6 & 25.2 & 26.7 & 31.1 \\
\hline Income and profits & 4.8 & 5.6 & 7.6 & 8.3 & 8.7 & 10.0 \\
\hline Goods and services & 4.6 & 6.5 & 8.4 & 9.0 & 9.3 & 11.3 \\
\hline International trade and transactions & 6.2 & 7.3 & 7.6 & 7.8 & 8.7 & 9.7 \\
\hline Tax on logging & $\ldots$ & 3.6 & 3.4 & 3.9 & 5.1 & 6.0 \\
\hline Other revenue & 0.7 & 1.8 & 2.7 & 4.5 & 5.5 & 5.7 \\
\hline Stamp duty & 0.2 & 0.2 & 0.2 & 0.3 & 0.3 & 0.3 \\
\hline Licenses and fees & 0.2 & 0.1 & 0.3 & 0.2 & 0.2 & 0.2 \\
\hline Miscellaneous revenue & 0.3 & 1.5 & 2.2 & 4.0 & 5.1 & 5.2 \\
\hline Grants & 2.7 & 18.0 & 21.8 & 36.6 & 32.5 & 32.2 \\
\hline Development grants 1/ & 2.2 & 14.2 & 17.3 & 34.8 & 29.6 & 31.2 \\
\hline Recurrent budget grants & 0.5 & 3.8 & 4.4 & 1.8 & 2.9 & 1.0 \\
\hline Expenditure 2/ & 28.0 & 37.4 & 39.1 & 63.6 & 63.0 & 70.4 \\
\hline Recurrent expenditure & 24.8 & 21.9 & 21.6 & 26.7 & 30.7 & 33.0 \\
\hline Compensation of employees & 11.7 & 9.3 & 8.8 & 10.6 & 9.8 & 11.9 \\
\hline Goods and services & 4.8 & 5.8 & 7.8 & 11.1 & 16.4 & 16.7 \\
\hline Interest & 1.3 & 0.9 & 0.9 & 0.9 & 0.6 & 1.2 \\
\hline Grants to provinces & 0.9 & 2.0 & 2.5 & 1.5 & 1.9 & 1.9 \\
\hline Employer social benefits & 0.2 & 0.2 & 0.2 & 0.1 & 0.1 & 0.1 \\
\hline Other & 5.9 & 3.8 & 1.4 & 2.6 & 2.0 & 1.2 \\
\hline Development expenditure & 3.2 & 15.5 & 17.4 & 36.9 & 32.3 & 37.5 \\
\hline Grant financed 1/ & 2.2 & 14.2 & 17.3 & 34.8 & 29.6 & 31.2 \\
\hline Concessional loan financed & 1.0 & 1.4 & 0.1 & 2.1 & 2.2 & 3.6 \\
\hline Domestically financed & 0.0 & 0.0 & 0.0 & 0.1 & 0.5 & 2.6 \\
\hline Recurrent balance (above the line) $3 /$ & -8.0 & 3.2 & 9.1 & 4.8 & 4.5 & 4.8 \\
\hline Primary domestic balance (above the line) 4/ & -8.2 & -1.2 & 5.5 & 1.7 & -0.6 & -1.2 \\
\hline Overall balance (above the line) & -9.0 & 1.8 & 9.0 & 2.6 & 1.7 & -1.5 \\
\hline Discrepancy (neg. are net expenditures) & -2.1 & -3.3 & 0.0 & -2.0 & 1.8 & 0.1 \\
\hline Overall balance (below the line) & -11.1 & -1.4 & 9.0 & 0.6 & -0.1 & -1.6 \\
\hline Financing & 11.1 & 1.4 & -9.0 & -0.6 & 0.1 & 1.6 \\
\hline Foreign (net) & 5.1 & 0.8 & 0.0 & 4.7 & 2.1 & 3.2 \\
\hline Disbursements & 5.5 & 1.3 & 0.1 & 2.1 & 2.2 & 3.6 \\
\hline Amortization (accrual) & 0.4 & 1.0 & 0.7 & 0.6 & 1.0 & 2.4 \\
\hline Debt forgiveness & 0.0 & 0.4 & 0.6 & 3.2 & 0.9 & 2.0 \\
\hline Domestic bank and nonbank (net) & -3.0 & -9.3 & -6.7 & -2.3 & -1.8 & -1.1 \\
\hline Banking system (accrual) & -1.1 & -9.1 & -8.7 & -1.9 & -1.9 & -0.7 \\
\hline Central bank & 2.5 & -3.8 & -5.8 & -2.8 & 0.0 & -1.3 \\
\hline Commercial banks & -3.5 & -5.3 & -2.9 & 0.9 & -1.9 & 0.6 \\
\hline Nonbank (accrual) & -1.9 & -0.2 & 2.0 & -0.4 & 0.1 & -0.5 \\
\hline National Provident Fund & -1.2 & 0.0 & 1.6 & -0.2 & -0.1 & -0.2 \\
\hline Other & -0.7 & -0.2 & 0.3 & -0.2 & 0.2 & -0.3 \\
\hline Privatization receipts & 0.0 & 0.0 & 0.0 & 0.0 & 0.3 & 0.0 \\
\hline Increase in expenditure arrears $5 /$ & 3.7 & 4.1 & -5.2 & -3.4 & -0.6 & -0.5 \\
\hline Principal debt arrears & 5.3 & 5.8 & -10.0 & 0.4 & 0.0 & 0.0 \\
\hline External & 1.9 & 0.1 & 0.5 & 0.4 & 0.0 & -3.2 \\
\hline Domestic & 3.4 & 5.7 & -10.5 & 0.0 & 0.0 & 0.0 \\
\hline Restructured Bonds & 0.0 & 0.0 & 12.8 & 0.0 & 0.0 & 0.0 \\
\hline \multicolumn{7}{|l|}{ Memorandum item: } \\
\hline Nominal GDP (in millions of S.I. dollars) & 1,517 & 1,718 & 1,961 & 2,207 & 2,542 & 2,971 \\
\hline
\end{tabular}

Sources: Data provided by the authorities; and Fund staff estimates.

1/ Estimated by staff through 2004. Data for 2005-2007 are from the authorities. Development grants and grant-financed development spending are currently administered by donors, and hence are not under the direct control of the government. 2/ On an accrual basis.

$3 /$ Includes domestic revenue, recurrent expenditure, and recurrent budget grant support.

4/ Defined as domestic revenue net of recurrent noninterest expenditure and nongrant financed development spending. $5 /$ Includes interest arrears. 
Table 6. Solomon Islands: Central Government Revenue and Grants, 2002-07

(In millions of Solomon Islands dollars)

\begin{tabular}{|c|c|c|c|c|c|c|}
\hline & 2002 & 2003 & 2004 & 2005 & 2006 & $\begin{array}{r}\text { Est. } \\
2007\end{array}$ \\
\hline Total revenue and grants & 287.6 & 673.5 & 943.0 & $1,462.3$ & $1,646.0$ & $2,049.2$ \\
\hline Total revenue & 247.2 & 364.0 & 515.9 & 655.0 & 820.4 & 1093.1 \\
\hline Tax revenue & 237.1 & 332.7 & 462.4 & 556.1 & 679.4 & 923.6 \\
\hline Income and profits & 73.2 & 96.1 & 148.4 & 183.9 & 221.3 & 297.8 \\
\hline Individuals & 37.6 & 50.5 & 60.7 & 93.8 & 109.3 & 145.3 \\
\hline Corporations & 35.6 & 45.6 & 87.7 & 90.1 & 112 & 152.5 \\
\hline Goods and services & 70.1 & 112.0 & 164.0 & 199.7 & 236.8 & 337.1 \\
\hline Sales taxes & 63.0 & 96.7 & 143.3 & 171.4 & 193.4 & 284.4 \\
\hline Excises & 7.1 & 15.3 & 20.7 & 28.3 & 43.4 & 52.7 \\
\hline International trade & 93.8 & 124.6 & 150.0 & 172.5 & 221.3 & 288.7 \\
\hline Customs and import duties & 54.5 & 67.8 & 82.4 & 84.5 & 87.7 & 105.1 \\
\hline Export taxes & 39.3 & 56.8 & 67.6 & 88.0 & 133.6 & 183.6 \\
\hline Other revenue & 10.1 & 31.3 & 53.5 & 98.9 & 141.0 & 169.5 \\
\hline Stamp duty & 3.0 & 3.7 & 4.3 & 5.7 & 7.3 & 9.5 \\
\hline Licenses and fees & 2.9 & 2.5 & 5.2 & 5.1 & 5.2 & 5.7 \\
\hline Miscellaneous revenue & 4.2 & 25.1 & 44.0 & 88.1 & 128.5 & 154.3 \\
\hline Grants & 40.4 & 309.5 & 427.1 & 807.3 & 825.6 & 956.1 \\
\hline Development grants $1 /$ & 33.3 & 243.7 & 340.0 & 767.3 & 751.4 & 927.5 \\
\hline Recurrent budget grants & 7.1 & 65.8 & 87.1 & 40.0 & 74.2 & 28.6 \\
\hline
\end{tabular}

Sources: Data provided by the Solomon Islands authorities; and Fund staff estimates.

1/ Estimated by staff through 2004. Data for 2005-2007 are from the authorities. Development grants and grant-financed development spending are currently administered by donors, and hence are not under the direct control of the government. 
Table 7. Solomon Islands: Central Government Expenditure, 2002-07

(In millions of Solomon Islands dollars)

\begin{tabular}{|c|c|c|c|c|c|c|}
\hline & 2002 & 2003 & 2004 & 2005 & 2006 & $\begin{array}{r}\text { Est. } \\
2007\end{array}$ \\
\hline Expenditure 1/ & 424.8 & 642.4 & 766.2 & $1,404.2$ & $1,602.1$ & $2,092.6$ \\
\hline Recurrent expenditure & 376.1 & 375.5 & 424.0 & 589.8 & 781.1 & 979.3 \\
\hline Compensation of employees & 177.4 & 160.2 & 172.9 & 232.9 & 249.8 & 354.1 \\
\hline Salaries and wages & 158.4 & 135.8 & 159.7 & 217.8 & 233.6 & 327.5 \\
\hline Of which: Arrears & -1.4 & -1.4 & 0.0 & 0.0 & 0.0 & 0.0 \\
\hline Social contributions & 18.9 & 24.4 & 13.2 & 15.1 & 16.2 & 26.6 \\
\hline Of which: Arrears & 9.3 & 9.3 & 0.0 & 0.0 & 0.0 & 0.0 \\
\hline Goods and services & 72.3 & 99.0 & 153.1 & 245.3 & 416.7 & 496.6 \\
\hline Of which: Arrears & 30.0 & 30.0 & 0.0 & 0.0 & 0.0 & 0.0 \\
\hline Realized contigent liabilities & 0.0 & 0.0 & 0.0 & 31.8 & 23.9 & 22.6 \\
\hline Interest & 20.4 & 14.6 & 17.8 & 20.4 & 15.0 & 36.3 \\
\hline To nonresidents & 2.3 & 4.8 & 6.7 & 7.6 & 2.5 & 24.0 \\
\hline Of which: Arrears & 14.2 & 19.1 & 4.9 & -23.5 & 6.1 & -6.7 \\
\hline To residents other than government & 18.1 & 9.9 & 6.5 & 7.9 & 9.3 & 9.3 \\
\hline Of which: Arrears & 0.0 & 0.0 & 1.9 & 5.9 & 2.8 & 5.6 \\
\hline To CBSI & 0.0 & 0.0 & 4.7 & 4.9 & 3.2 & 3.0 \\
\hline Grants & 14.2 & 37.5 & 52.5 & 35.3 & 50.8 & 59.8 \\
\hline To international organizations & 0.0 & 3.3 & 3.2 & 2.6 & 1.3 & 2.5 \\
\hline To other government units & 14.2 & 34.2 & 49.3 & 32.7 & 49.5 & 57.4 \\
\hline Of which: Arrears & 0.0 & 9.5 & 0.0 & 0.0 & 0.0 & 0.0 \\
\hline Employer social benefits & 2.8 & 3.0 & 3.0 & 3.0 & 3.0 & 3.0 \\
\hline Other expenses & 89.0 & 59.0 & 22.5 & 18.7 & 19.3 & 2.7 \\
\hline Property expense other than interest & 0.3 & 1.8 & 1.8 & 2.0 & 2.3 & 2.7 \\
\hline Miscellaneous other expenses $2 /$ & 88.7 & 57.2 & 20.7 & 16.7 & 17.0 & 0.0 \\
\hline Nonfinancial assets & 0.0 & 2.3 & 2.3 & 2.3 & 2.6 & 4.3 \\
\hline Purchase & 0.0 & 2.3 & 2.3 & 2.3 & 2.6 & 4.3 \\
\hline Sale & 0.0 & 0.0 & 0.0 & 0.0 & 0.0 & 0.0 \\
\hline Development expenditure & 48.7 & 266.9 & 342.1 & 814.4 & 821.04 & 1113.3 \\
\hline Grant financed 3/ & 33.3 & 243.7 & 340.0 & 767.3 & 751.4 & 927.5 \\
\hline Of which: Cash & 20.8 & 152.3 & 170.0 & 450.7 & 431.1 & 583.1 \\
\hline Loan financed & 15.4 & 23.2 & 2.1 & 47.1 & 69.7 & 185.8 \\
\hline Domestic & 0.0 & 0.0 & 0.0 & 1.8 & 13.9 & 77.8 \\
\hline Foreign & 15.4 & 23.2 & 2.1 & 45.3 & 55.8 & 108.0 \\
\hline Recurrent balance & -121.8 & 54.3 & 179.0 & 105.2 & 113.5 & 142.4 \\
\hline Overall balance (above-the-line) & -137.2 & 31.1 & 176.8 & 58.1 & 43.8 & -43.4 \\
\hline
\end{tabular}

Sources: Data provided by the Solomon Islands authorities; and Fund staff estimates.

1/ All expenditures are presented on an accrual basis.

2/ Including compensation payments.

3/ Estimated by staff through 2004. Data for 2005-2007 are from the authorities. Development grants and grant-financed development spending are currently administered by donors, and hence are not under the direct control of the government. 
Table 8. Solomon Islands: Central Government Budget Financing, 2002-07 1/

(In millions of Solomon Islands dollars)

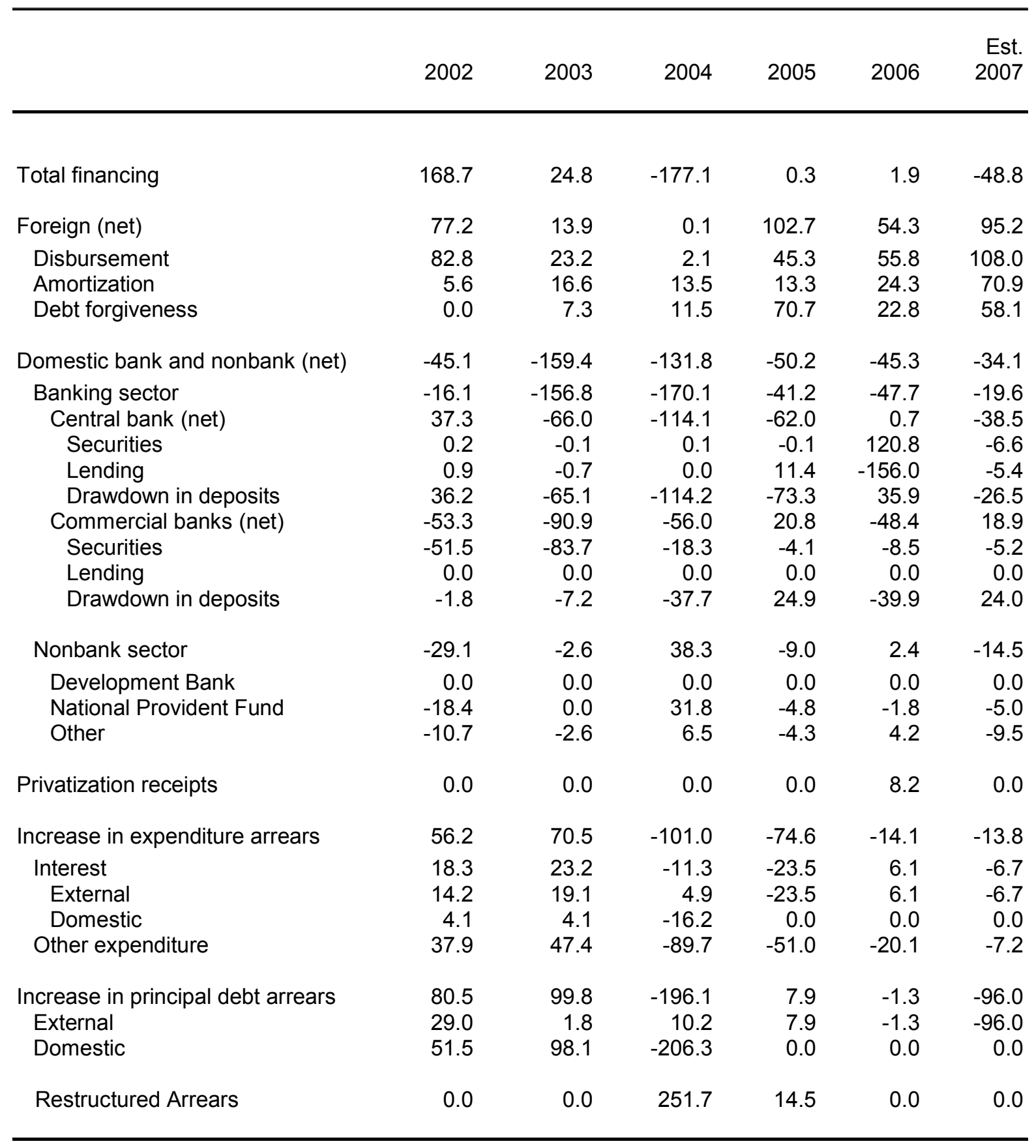

Sources: Data provided by the Solomon Islands authorities; and Fund staff estimates.

1/ All debt servicing is presented on an accrual basis. 
Table 9. Solomon Islands: Central Government Gross Domestic Debt, 2002-07

\begin{tabular}{|c|c|c|c|c|c|c|}
\hline & 2002 & 2003 & 2004 & 2005 & 2006 & $\begin{array}{r}\text { Est. } \\
2007\end{array}$ \\
\hline & \multicolumn{6}{|c|}{ (In millions of Solomon Islands dollars) } \\
\hline Total & 452.9 & 463.8 & 484.0 & 482.4 & 440.8 & 409.1 \\
\hline Securities & 277.9 & 289.5 & 309.6 & 296.7 & 411.1 & 384.8 \\
\hline Bonds & 245.6 & 245.6 & 269.4 & 258.0 & 249.1 & 237.2 \\
\hline Bills & 21.2 & 32.8 & 29.1 & 27.6 & 30.7 & 22.4 \\
\hline Other & 11.1 & 11.1 & 11.1 & 11.1 & 131.3 & 125.3 \\
\hline \multirow[t]{2}{*}{ Loans } & 175.0 & 174.3 & 174.4 & 185.7 & 29.7 & 24.32 \\
\hline & \multicolumn{6}{|c|}{ (Distribution by holder) } \\
\hline Central bank & 186.3 & 185.5 & 185.6 & 196.9 & 161.7 & 149.7 \\
\hline Securities & 11.3 & 11.2 & 11.3 & 11.2 & 132.0 & 125.4 \\
\hline Bonds & 0.0 & 0.0 & 0.0 & 0.0 & 0.0 & 0.0 \\
\hline Bills & 0.2 & 0.1 & 0.2 & 0.1 & 0.6 & 0.1 \\
\hline Other & 11.1 & 11.1 & 11.1 & 11.1 & 131.3 & 125.3 \\
\hline Loans & 175.0 & 174.3 & 174.3 & 185.7 & 29.7 & 24.3 \\
\hline Commercial banks & 149.8 & 164.2 & 145.9 & 141.8 & 133.3 & 128.1 \\
\hline Securities & 149.8 & 164.2 & 145.9 & 141.8 & 133.3 & 128.1 \\
\hline Bonds & 149.8 & 149.8 & 141.9 & 135.1 & 128.3 & 121.4 \\
\hline Bills & 0.0 & 14.4 & 4.0 & 6.7 & 5.0 & 6.7 \\
\hline Other & 0.0 & 0.0 & 0.0 & 0.0 & 0.0 & 0.0 \\
\hline Loans & 0.0 & 0.0 & 0.0 & 0.0 & 0.0 & 0.0 \\
\hline National Provident Fund & 94.3 & 94.3 & 126.1 & 121.6 & 119.5 & 114.6 \\
\hline Securities & 94.3 & 94.3 & 126.1 & 121.6 & 119.5 & 114.6 \\
\hline Bonds & 94.3 & 94.3 & 126.1 & 120.5 & 115.2 & 107.6 \\
\hline Bills & 0.0 & 0.0 & 0.0 & 1.1 & 4.3 & 6.9 \\
\hline Other & 0.0 & 0.0 & 0.0 & 0.0 & 0.0 & 0.0 \\
\hline Loans & 0.0 & 0.0 & 0.0 & 0.0 & 0.0 & 0.0 \\
\hline Statutory corporations & 0.0 & 0.0 & 1.3 & 0.0 & 0.5 & 0.0 \\
\hline Securities & 0.0 & 0.0 & 1.2 & 0.0 & 0.5 & 0.0 \\
\hline Bonds & 0.0 & 0.0 & 0.0 & 0.0 & 0.0 & 0.0 \\
\hline Bills & 0.0 & 0.0 & 1.2 & 0.0 & 0.5 & 0.0 \\
\hline Other & 0.0 & 0.0 & 0.0 & 0.0 & 0.0 & 0.0 \\
\hline Loans & 0.0 & 0.0 & 0.1 & 0.0 & 0.0 & 0.0 \\
\hline Others & 22.5 & 19.9 & 26.4 & 22.1 & 26.3 & 16.8 \\
\hline Securities & 22.5 & 19.9 & 26.4 & 22.1 & 26.3 & 16.8 \\
\hline Bonds & 1.5 & 1.5 & 1.4 & 1.3 & 1.3 & 1.2 \\
\hline Bills & 21.0 & 18.4 & 25.0 & 20.8 & 25.1 & 15.6 \\
\hline Other & $\ldots$ & $\ldots$ & $\ldots$ & $\ldots$ & $\ldots$ & $\ldots$ \\
\hline \multirow[t]{2}{*}{ Loans } & $\ldots$ & $\ldots$ & $\ldots$ & $\ldots$ & $\ldots$ & $\ldots$ \\
\hline & \multicolumn{6}{|c|}{ (In percent of total) } \\
\hline Central bank & 41.1 & 40.0 & 38.3 & 40.8 & 36.7 & 36.6 \\
\hline Securities & 2.5 & 2.4 & 2.3 & 2.3 & 29.9 & 30.6 \\
\hline Bonds & 0.0 & 0.0 & 0.0 & 0.0 & 0.0 & 0.0 \\
\hline Bills & 0.0 & 0.0 & 0.0 & 0.0 & 0.1 & 0.0 \\
\hline Other & 2.4 & 2.4 & 2.3 & 2.3 & 29.8 & 30.6 \\
\hline Loans & 38.6 & 37.6 & 36.0 & 38.5 & 6.7 & 5.9 \\
\hline Commercial banks & 33.1 & 35.4 & 30.1 & 29.4 & 30.2 & 31.3 \\
\hline Securities & 33.1 & 35.4 & 30.1 & 29.4 & 30.2 & 31.3 \\
\hline Bonds & 33.1 & 32.3 & 29.3 & 28.0 & 29.1 & 29.7 \\
\hline Bills & 0.0 & 3.1 & 0.8 & 1.4 & 1.1 & 1.6 \\
\hline Other & 0.0 & 0.0 & 0.0 & 0.0 & 0.0 & 0.0 \\
\hline Loans & 0.0 & 0.0 & 0.0 & 0.0 & 0.0 & 0.0 \\
\hline National Provident Fund & 20.8 & 20.3 & 26.1 & 25.2 & 27.1 & 28.0 \\
\hline Securities & 20.8 & 20.3 & 26.1 & 25.2 & 27.1 & 28.0 \\
\hline Bonds & 20.8 & 20.3 & 26.1 & 25.0 & 26.1 & 26.3 \\
\hline Bills & 0.0 & 0.0 & 0.0 & 0.2 & 1.0 & 1.7 \\
\hline Other & 0.0 & 0.0 & 0.0 & 0.0 & 0.0 & 0.0 \\
\hline Loans & 0.0 & 0.0 & 0.0 & 0.0 & 0.0 & 0.0 \\
\hline Others & 5.0 & 4.3 & 5.4 & 4.6 & 6.0 & 4.1 \\
\hline Securities & 5.0 & 4.3 & 5.4 & 4.6 & 6.0 & 4.1 \\
\hline Bonds & 0.3 & 0.3 & 0.3 & 0.3 & 0.3 & 0.3 \\
\hline Bills & 4.6 & 4.0 & 5.2 & 4.3 & 5.7 & 3.8 \\
\hline Other & $\ldots$ & $\ldots$ & $\ldots$ & $\ldots$ & $\ldots$ & $\ldots$ \\
\hline
\end{tabular}

Sources: Central Bank of Solomon Islands; Ministry of Finance; and Fund staff estimates. 
Table 10. Solomon Islands: Equity Investment of the Investment Corporation of Solomon Islands, 2003-07

(Percent share)

\begin{tabular}{|c|c|c|c|c|c|}
\hline & 2003 & 2004 & 2005 & 2006 & 2007 \\
\hline \multicolumn{6}{|l|}{ Nonfinancial sector } \\
\hline \multicolumn{6}{|l|}{ Agriculture/agro-industry sectors } \\
\hline Solomon Islands Plantation Ltd. & 30 & 30 & 98 & 98 & $\ldots$ \\
\hline Kolombangara Forestry and Plantation Ltd. & 19 & 19 & 40 & 40 & $\ldots$ \\
\hline Solomon Taiyo Ltd. & $\ldots$ & $\ldots$ & $\ldots$ & $\ldots$ & $\ldots$ \\
\hline Soltai Fishing Company 1/ & 51 & 51 & 51 & 51 & $\ldots$ \\
\hline \multicolumn{6}{|l|}{ Others } \\
\hline Sasape Marina Ltd. & 100 & 100 & 100 & 100 & $\ldots$ \\
\hline Solomon Airlines & 100 & 100 & 100 & 100 & $\ldots$ \\
\hline Solomon Telekom & 7 & 7 & 3 & 3 & $\ldots$ \\
\hline Pacific Forum Line Ltd. & $\ldots$ & $\ldots$ & 0.6 & 0.6 & $\ldots$ \\
\hline Solomon Islands Printers & 100 & 100 & 100 & 100 & $\ldots$ \\
\hline \multicolumn{6}{|l|}{ Financial sector } \\
\hline Development Bank of Solomon Islands & 10 & 10 & 10 & 10 & $\ldots$ \\
\hline Central Bank of Solomon Islands & 100 & 100 & 100 & 100 & $\ldots$ \\
\hline
\end{tabular}

Source: Central Bank of Solomon Islands Annual Report .

1/ Incorporated to take over Solomon Taiyo's assets. The remaining 49 percent is owned by the Western Province government. 
Table 11. Solomon Islands: Summary Accounts of the Banking System, 2004-2007 (In millons of Solomon Islands dollars, unless otherwise specified)

\begin{tabular}{|c|c|c|c|c|}
\hline & 2004 & 2005 & 2006 & 2007 \\
\hline \multicolumn{5}{|l|}{ I. Central Bank } \\
\hline $\begin{array}{l}\text { Net foreign reserves } \\
\text { Assets } \\
\text { Liabilities }\end{array}$ & $\begin{array}{l}578.2 \\
600.7 \\
-22.5\end{array}$ & $\begin{array}{l}699.7 \\
716.9 \\
-17.2\end{array}$ & $\begin{array}{l}773.1 \\
790.5 \\
-17.4\end{array}$ & $\begin{array}{l}873.5 \\
918.0 \\
-44.5\end{array}$ \\
\hline $\begin{array}{l}\text { Net domestic assets } \\
\text { Net credit to central government } \\
\text { Credit } \\
\text { Deposits } \\
\text { Credit to rest of NFPS } \\
\text { Other items (net) }\end{array}$ & $\begin{array}{r}-194.6 \\
-20.5 \\
185.6 \\
-206.1 \\
0.0 \\
-174.0\end{array}$ & $\begin{array}{r}-237.2 \\
-82.5 \\
196.9 \\
-279.4 \\
0.0 \\
-154.6\end{array}$ & $\begin{array}{r}-282.5 \\
-81.9 \\
161.7 \\
-243.5 \\
0.0 \\
-200.6\end{array}$ & $\begin{array}{r}-398.3 \\
-120.3 \\
149.7 \\
-270.0 \\
0.0 \\
-278.0\end{array}$ \\
\hline $\begin{array}{l}\text { Reserve money } \\
\text { Currency outside banks } \\
\text { Banking system reserves } \\
\text { Currency in vault } \\
\text { Deposits at CBSI }\end{array}$ & $\begin{array}{r}383.6 \\
123.2 \\
260.4 \\
14.5 \\
245.9\end{array}$ & $\begin{array}{r}462.6 \\
153.0 \\
309.5 \\
15.0 \\
294.6\end{array}$ & $\begin{array}{r}490.6 \\
179.3 \\
311.3 \\
24.1 \\
287.2\end{array}$ & $\begin{array}{r}475.2 \\
234.6 \\
240.6 \\
28.4 \\
212.3\end{array}$ \\
\hline \multicolumn{5}{|l|}{ II. Commercial banks } \\
\hline Net foreign assets & 5.7 & 12.8 & -3.1 & -21.5 \\
\hline Monetary reserves and $\mathrm{CBSI}$ securities & 260.4 & 309.5 & 311.3 & 240.6 \\
\hline $\begin{array}{l}\text { Net domestic assets } \\
\text { Net credit to central government } \\
\text { Credit } \\
\text { Deposits } \\
\text { Net credit to local government } \\
\text { Credit } \\
\text { Deposits } \\
\text { Credit to rest of NFPS }\end{array}$ & $\begin{array}{r}287.7 \\
106.3 \\
149.8 \\
-43.5 \\
-3.9 \\
0.2 \\
-4.1 \\
-21.3\end{array}$ & $\begin{array}{r}389.2 \\
127.4 \\
146.0 \\
-18.6 \\
-4.2 \\
0.0 \\
-4.2 \\
-18.2\end{array}$ & $\begin{array}{r}595.2 \\
80.8 \\
138.3 \\
-57.6 \\
-5.1 \\
0.0 \\
-5.1 \\
-52.2\end{array}$ & $\begin{array}{r}886.6 \\
93.6 \\
130.1 \\
-36.6 \\
-2.0 \\
0.0 \\
-2.0 \\
-46.7\end{array}$ \\
\hline Credit to private sector & 238.6 & 389.1 & 617.2 & 956.7 \\
\hline Other items (net) & -31.9 & -104.9 & -45.4 & -114.9 \\
\hline Deposits & 553.8 & 711.5 & 903.5 & $1,105.7$ \\
\hline \multicolumn{5}{|l|}{ III. Banking System } \\
\hline Net foreign assets & 583.9 & 712.5 & 770.1 & 852.1 \\
\hline $\begin{array}{l}\text { Net domestic assets } \\
\text { Net domestic credit } \\
\text { Net credit to central government } \\
\text { Credit }\end{array}$ & $\begin{array}{r}93.2 \\
299.1 \\
85.7 \\
335.4\end{array}$ & $\begin{array}{r}152.0 \\
411.5 \\
44.8 \\
342.9\end{array}$ & $\begin{array}{r}312.7 \\
558.8 \\
-1.1 \\
300.0\end{array}$ & $\begin{array}{r}488.3 \\
881.2 \\
-26.8 \\
279.8\end{array}$ \\
\hline Deposits & -249.6 & -298.0 & -301.1 & -306.6 \\
\hline Net credit to local government & -3.9 & -4.2 & -5.1 & -2.0 \\
\hline Credit & 0.2 & 0.0 & 0.0 & 0.0 \\
\hline Deposits & -4.1 & -4.2 & -5.1 & -2.0 \\
\hline Credit to rest of NFPS & -21.3 & -18.2 & -52.2 & -46.7 \\
\hline Credit to private sector & 238.6 & 389.1 & 617.2 & 956.7 \\
\hline Other items (net) & -205.9 & -259.5 & -246.1 & -392.9 \\
\hline $\begin{array}{l}\text { M3 } \\
\text { Narrow money }\end{array}$ & $\begin{array}{l}621.7 \\
371.8\end{array}$ & $\begin{array}{l}863.7 \\
537.9\end{array}$ & $\begin{array}{r}1,091.6 \\
703.4\end{array}$ & $\begin{array}{r}1,353.0 \\
909.7\end{array}$ \\
\hline Currency outside banks & 123.2 & 153.0 & 177.6 & 232.0 \\
\hline Demand deposits & 248.6 & 384.9 & 525.8 & 677.7 \\
\hline Quasi-money & 249.8 & 325.8 & 388.1 & 443.3 \\
\hline Savings deposits & 76.0 & 88.1 & 98.9 & 122.4 \\
\hline Time deposits & 173.9 & 237.8 & 289.2 & 320.9 \\
\hline
\end{tabular}

Source: Central Bank of Solomon Islands. 
Table 12. Solomon Islands: Assets and Liabilities of the Central Bank of Solomon Islands, 2004-07

(In millions of Solomon Islands dollars, end of period)

\begin{tabular}{lrrrr}
\hline & & & & Est. \\
& 2004 & 2005 & 2006 & 2007 \\
& & & & \\
\hline Assets & & & & \\
External & 806.0 & 942.2 & $1,004.0$ & $1,143.1$ \\
Domestic & 596.8 & 716.9 & 790.5 & 918.0 \\
$\quad$ Central government & 209.2 & 225.3 & 213.5 & 225.0 \\
$\quad$ Loans & 185.6 & 196.9 & 161.7 & 149.7 \\
$\quad 174.3$ & 185.7 & 29.7 & 24.3 \\
Securities & 11.3 & 11.2 & 132.0 & 125.4 \\
$\quad$ Securities and investments & 23.7 & 28.4 & 51.8 & 75.4 \\
$\quad$ Other & 0.7 & 0.0 & 0.0 & 0.0 \\
& 23.0 & 28.4 & 51.8 & 75.4 \\
Liabilities & & & & \\
External & 806.0 & 942.2 & $1,104.0$ & $1,143.1$ \\
Domestic & 22.5 & 17.2 & 71.4 & 44.5 \\
Currency issued & 783.5 & 925.0 & $1,032.6$ & $1,098.6$ \\
Deposits & 137.7 & 168.0 & 203.4 & 262.9 \\
$\quad$ Banks & 451.9 & 575.2 & 534.8 & 475.9 \\
$\quad$ Central government & 244.6 & 294.7 & 287.1 & 200.7 \\
$\quad$ Other & 206.1 & 279.4 & 243.5 & 270.0 \\
Central bank bills & 1.2 & 1.1 & 4.2 & 5.1 \\
Other (including provisions) & 0.0 & 0.0 & 0.0 & 0.0 \\
Capital & 177.6 & 23.2 & 135.8 & 150.6 \\
& 16.3 & 158.6 & 158.6 & 209.1 \\
\hline
\end{tabular}

Source: Central Bank of Solomon Islands. 
Table 13. Solomon Islands: Assets and Liabilities of Commercial Banks, 2004-07

(In millions of Solomon Islands dollars, end of period)

\begin{tabular}{|c|c|c|c|c|}
\hline & 2004 & 2005 & 2006 & $\begin{array}{r}\text { Est. } \\
2007\end{array}$ \\
\hline Assets & 775.6 & 990.6 & $1,273.1$ & $1,505.3$ \\
\hline External & 71.9 & 75.6 & 79.4 & 62.8 \\
\hline Domestic & 703.7 & 915.0 & $1,193.7$ & $1,442.5$ \\
\hline Cash & 14.5 & 15.0 & 24.1 & 28.4 \\
\hline Deposits at central bank & 245.9 & 294.6 & 287.2 & 212.3 \\
\hline Securities & 149.8 & 141.8 & 133.3 & 128.1 \\
\hline Treasury bills & 7.9 & 6.7 & 5.0 & 6.7 \\
\hline Other government securities & 141.9 & 135.1 & 128.3 & 121.4 \\
\hline Central bank securities (Bokolo bills) & 0.0 & 0.0 & 0.0 & 0.0 \\
\hline Loans and advances & 234.9 & 389.1 & 617.2 & 956.7 \\
\hline Central government & 0.0 & 0.0 & 0.0 & 0.0 \\
\hline Provincial governments & 0.2 & 0.0 & 0.0 & 0.0 \\
\hline Statutory corporations & 0.0 & 0.0 & 0.0 & 0.0 \\
\hline Other & 234.7 & 389.1 & 617.2 & 956.7 \\
\hline Premises, equipment, and other & 58.6 & 74.6 & 131.9 & 117.1 \\
\hline Liabilities & 775.6 & 990.6 & $1,273.1$ & $1,505.3$ \\
\hline External & 66.2 & 62.8 & 82.5 & 84.2 \\
\hline Domestic & 709.4 & 927.9 & $1,190.6$ & $1,421.0$ \\
\hline Deposits & 544.9 & 732.4 & 966.1 & $1,144.3$ \\
\hline Central government & 43.5 & 18.6 & 57.6 & 36.6 \\
\hline Provincial governments & 4.1 & 4.2 & 5.1 & 2.0 \\
\hline Statutory corporations & 21.3 & 18.3 & 52.2 & 46.8 \\
\hline Other & 475.9 & 691.3 & 851.3 & $1,059.0$ \\
\hline Capital and reserves & 121.1 & 159.2 & 180.2 & 188.3 \\
\hline Other & 43.4 & 36.3 & 44.3 & 88.3 \\
\hline
\end{tabular}

Source: Central Bank of Solomon Islands. 
Table 14a. Solomon Islands: Commercial Bank Advances and Loans, 2004-08

(In millions of Solomon Islands dollars)

\begin{tabular}{|c|c|c|c|c|c|c|c|c|c|c|}
\hline End of Period & Agriculture & Forestry & Fisheries & $\begin{array}{l}\text { Mining \& } \\
\text { Quarrying }\end{array}$ & Manufacturing & Construction & Transport & $\begin{array}{l}\text { Telecom- } \\
\text { munications }\end{array}$ & Distribution & Tourism \\
\hline 2004 & 4.5 & 42.1 & 0.3 & 0.1 & 26.8 & 14.9 & 2.0 & 42.8 & 46.9 & 2.6 \\
\hline 2005 & 4.4 & 60.5 & 9.6 & 0.3 & 60.0 & 21.1 & 5.4 & 60.5 & 90.1 & 2.5 \\
\hline 2006 & 8.2 & 86.8 & 22.6 & 0.0 & 61.0 & 42.3 & 18.7 & 82.2 & 103.0 & 15.7 \\
\hline 2007 & 88.7 & 55.5 & 24.6 & 0.1 & 122.1 & 60.2 & 28.1 & 119.0 & 129.4 & 43.9 \\
\hline \multicolumn{11}{|l|}{2006} \\
\hline January & 4.5 & 63.4 & 9.5 & 0.3 & 58.0 & 19.9 & 5.5 & 59.8 & 88.7 & 2.8 \\
\hline February & 5.5 & 67.9 & 5.4 & 0.3 & 71.3 & 18.7 & 5.1 & 59.2 & 89.7 & 2.8 \\
\hline March & 4.6 & 78.6 & 7.4 & 0.3 & 55.8 & 20.9 & 4.7 & 58.5 & 88.7 & 2.8 \\
\hline April & 4.1 & 82.8 & 8.1 & 0.3 & 50.4 & 21.5 & 5.3 & 59.5 & 90.0 & 2.7 \\
\hline May & 4.6 & 90.6 & 13.2 & 0.3 & 45.0 & 22.2 & 4.4 & 62.4 & 93.9 & 3.7 \\
\hline June & 6.5 & 86.8 & 14.4 & 0.0 & 53.8 & 23.3 & 4.9 & 59.8 & 97.7 & 4.1 \\
\hline July & 7.7 & 92.4 & 18.5 & 0.0 & 50.6 & 31.2 & 5.4 & 74.1 & 90.1 & 5.0 \\
\hline August & 8.5 & 94.7 & 14.9 & 0.0 & 56.0 & 34.5 & 5.1 & 74.8 & 90.7 & 5.8 \\
\hline September & 7.5 & 93.8 & 19.2 & 0.0 & 69.5 & 39.9 & 7.2 & 77.7 & 87.5 & 6.6 \\
\hline October & 7.7 & 80.4 & 15.0 & 0.0 & 55.3 & 36.7 & 7.0 & 80.3 & 90.5 & 8.0 \\
\hline November & 8.2 & 100.3 & 21.3 & 0.0 & 56.1 & 37.4 & 7.1 & 80.3 & 93.3 & 14.1 \\
\hline December & 8.2 & 86.8 & 22.6 & 0.0 & 61.0 & 42.3 & 18.7 & 82.2 & 103.0 & 15.7 \\
\hline \multicolumn{11}{|l|}{2007} \\
\hline January & 8.4 & 77.1 & 24.3 & 0.0 & 59.7 & 44.0 & 20.6 & 80.7 & 100.6 & 19.9 \\
\hline February & 7.7 & 88.2 & 24.9 & 0.0 & 70.6 & 45.3 & 24.2 & 73.3 & 100.7 & 20.8 \\
\hline March & 10.9 & 87.1 & 30.7 & 0.0 & 63.6 & 47.7 & 27.3 & 78.2 & 106.1 & 21.2 \\
\hline April & 10.2 & 93.1 & 29.8 & 0.0 & 43.5 & 45.6 & 30.3 & 75.5 & 103.2 & 27.3 \\
\hline May & 9.4 & 92.7 & 29.9 & 0.0 & 50.4 & 46.0 & 23.4 & 75.1 & 101.5 & 29.5 \\
\hline June & 13.0 & 108.8 & 31.2 & 0.0 & 72.2 & 48.8 & 21.9 & 78.3 & 110.7 & 30.7 \\
\hline July & 9.7 & 98.2 & 29.5 & 0.0 & 70.1 & 46.7 & 26.2 & 109.1 & 115.3 & 29.5 \\
\hline August & 9.7 & 109.8 & 33.9 & 0.2 & 98.0 & 45.3 & 30.6 & 109.1 & 122.6 & 39.7 \\
\hline September & 9.5 & 126.2 & 32.3 & 0.3 & 97.5 & 49.0 & 29.0 & 106.8 & 122.9 & 38.6 \\
\hline October & 13.1 & 113.7 & 24.4 & 0.5 & 91.1 & 54.9 & 26.9 & 105.4 & 126.8 & 43.6 \\
\hline November & 10.2 & 116.1 & 25.3 & 0.1 & 84.3 & 50.9 & 28.1 & 124.0 & 123.1 & 43.6 \\
\hline December & 88.7 & 55.5 & 24.6 & 0.1 & 122.1 & 60.2 & 28.1 & 119.0 & 129.4 & 43.9 \\
\hline \multicolumn{11}{|l|}{2008} \\
\hline January & 12.9 & 137.6 & 25.9 & 0.1 & 112.5 & 60.1 & 27.0 & 120.5 & 132.8 & 44.6 \\
\hline February & 11.5 & 132.3 & 26.1 & 0.1 & 121.5 & 66.4 & 27.9 & 134.9 & 134.6 & 46.7 \\
\hline March & 13.1 & 123.2 & 30.9 & 1.9 & 107.8 & 67.0 & 32.6 & 133.0 & 133.7 & 46.8 \\
\hline
\end{tabular}

Source: Central Bank of Solomon Islands. 
Table 14b. Solomon Islands: Commercial Bank Advances and Loans, 2004-08

(In millions of Solomon Islands dollars)

\begin{tabular}{|c|c|c|c|c|c|c|c|c|c|}
\hline End of Period & $\begin{array}{l}\text { Entertainment } \\
\text { \& Catering }\end{array}$ & Government & $\begin{array}{c}\text { Statutory } \\
\text { Corporations }\end{array}$ & $\begin{array}{l}\text { Public Financial } \\
\text { Institutions }\end{array}$ & $\begin{array}{l}\text { Private Financial } \\
\text { Institutions }\end{array}$ & $\begin{array}{l}\text { Professional \& } \\
\text { Other Services }\end{array}$ & Personal & Total & $\begin{array}{l}\text { Non- } \\
\text { Resident }\end{array}$ \\
\hline 2004 & 1.7 & 0.2 & 0.1 & 0.0 & 0.0 & 15.0 & 38.6 & 238.9 & 1.6 \\
\hline 2005 & 3.9 & 0.0 & 0.0 & 0.0 & 0.0 & 17.3 & 53.7 & 389.1 & 4.5 \\
\hline 2006 & 3.3 & 0.0 & 1.0 & 0.0 & 0.2 & 45.9 & 117.1 & 607.9 & 0.4 \\
\hline 2007 & 0.9 & 0.0 & 0.8 & 0.0 & 0.4 & 49.8 & 191.1 & 914.8 & 1.8 \\
\hline \multicolumn{10}{|l|}{2006} \\
\hline January & 4.1 & 0.0 & 0.2 & 0.0 & 0.0 & 18.8 & 64.6 & 400.1 & 0.4 \\
\hline February & 4.3 & 0.0 & 0.0 & 0.0 & 0.0 & 25.2 & 68.8 & 424.2 & 0.4 \\
\hline March & 4.4 & 0.0 & 0.3 & 0.3 & 0.3 & 27.7 & 76.9 & 432.2 & 0.4 \\
\hline April & 4.5 & 0.0 & 0.1 & 0.0 & 0.3 & 28.7 & 76.1 & 434.3 & 0.4 \\
\hline May & 4.4 & 0.1 & 0.0 & 0.0 & 0.3 & 31.3 & 79.9 & 456.2 & 0.4 \\
\hline June & 4.4 & 0.0 & 0.0 & 0.0 & 0.3 & 35.6 & 84.0 & 475.7 & 0.4 \\
\hline July & 4.5 & 0.0 & 0.1 & 0.0 & 0.3 & 36.2 & 87.3 & 503.4 & 0.4 \\
\hline August & 3.3 & 0.0 & 0.1 & 0.0 & 0.2 & 37.6 & 91.2 & 517.4 & 0.5 \\
\hline September & 3.2 & 0.0 & 0.4 & 0.0 & 0.3 & 44.0 & 91.9 & 548.7 & 0.4 \\
\hline October & 3.4 & 0.0 & 0.9 & 0.3 & 0.2 & 43.2 & 99.1 & 528.0 & 0.4 \\
\hline November & 3.6 & 0.0 & 0.9 & 0.2 & 0.2 & 43.4 & 107.0 & 573.5 & 0.4 \\
\hline December & 3.3 & 0.0 & 1.0 & 0.0 & 0.2 & 45.9 & 117.1 & 607.9 & 0.4 \\
\hline \multicolumn{10}{|l|}{2007} \\
\hline January & 4.2 & 0.0 & 1.0 & 0.0 & 0.3 & 45.5 & 122.3 & 608.5 & 0.4 \\
\hline February & 4.1 & 0.0 & 1.0 & 0.2 & 0.3 & 48.3 & 131.7 & 641.1 & 0.5 \\
\hline March & 4.3 & 1.6 & 0.9 & 0.8 & 0.3 & 54.4 & 134.9 & 669.9 & 0.5 \\
\hline April & 0.3 & 0.0 & 1.3 & 0.0 & 0.3 & 54.9 & 141.0 & 656.1 & 0.5 \\
\hline May & 0.4 & 0.0 & 0.6 & 0.0 & 0.3 & 53.1 & 143.4 & 655.7 & 0.4 \\
\hline June & 0.5 & 0.0 & 0.3 & 0.0 & 5.9 & 49.5 & 148.5 & 720.4 & 0.4 \\
\hline July & 0.6 & 0.0 & 0.3 & 0.0 & 0.3 & 48.5 & 156.0 & 740.0 & 0.4 \\
\hline August & 0.6 & 0.1 & 0.3 & 0.0 & 0.4 & 49.1 & 162.3 & 811.6 & 0.4 \\
\hline September & 0.6 & 0.0 & 0.7 & 0.0 & 0.3 & 48.9 & 163.7 & 826.2 & 0.4 \\
\hline October & 0.6 & 0.0 & 0.8 & 0.0 & 0.4 & 50.9 & 169.1 & 822.1 & 0.4 \\
\hline November & 0.7 & 0.0 & 0.5 & 0.0 & 0.3 & 51.3 & 181.6 & 840.3 & 1.9 \\
\hline December & 0.9 & 0.0 & 0.8 & 0.0 & 0.4 & 49.8 & 191.1 & 914.8 & 1.8 \\
\hline \multicolumn{10}{|l|}{2008} \\
\hline January & 0.8 & 0.0 & 1.1 & 0.0 & 0.3 & 49.4 & 195.1 & 920.8 & 1.9 \\
\hline February & 1.0 & 0.0 & 1.1 & 0.0 & 0.4 & 53.1 & 207.8 & 965.5 & 1.8 \\
\hline March & 1.1 & 0.0 & 1.3 & 0.0 & 1.0 & 51.8 & 209.9 & 954.9 & 1.8 \\
\hline
\end{tabular}

Source: Central Bank of Solomon Islands. 
Table 15. Solomon Islands: Commercial Bank Interest Rates on Savings and Time Deposits, 2004-08

(In percent, end of period)

\begin{tabular}{|c|c|c|c|c|c|c|c|}
\hline \multirow[b]{2}{*}{ End of Period } & \multirow[b]{2}{*}{$\begin{array}{c}\text { Savings } \\
\text { From }\end{array}$} & \multirow[b]{2}{*}{ Deposits } & \multicolumn{4}{|c|}{ Time Deposits } & \multirow[b]{2}{*}{$\begin{array}{l}\text { Weighted Average } \\
\text { Rate of Interest } \\
\text { On Deposits }\end{array}$} \\
\hline & & & Up to 3 Months & 3-6 Months & 6-12 Months & Over 12 Months & \\
\hline 2004 & 0.25 & -0.50 & $0.25-1.00$ & $0.50-1.25$ & $0.50-1.50$ & $0.00-2.00$ & 0.72 \\
\hline 2005 & 0.00 & -0.20 & $0.00-1.00$ & $0.25-1.25$ & $0.25-1.50$ & $0.00-2.25$ & 0.91 \\
\hline 2006 & 0.25 & -2.00 & $0.25-1.00$ & $0.25-1.25$ & $0.50-1.50$ & $0.00-2.25$ & 0.94 \\
\hline 2007 & 0.25 & -2.00 & $0.25-0.50$ & $0.25-0.75$ & $0.25-0.75$ & $0.50-1.43$ & 0.65 \\
\hline \multicolumn{8}{|l|}{2004} \\
\hline January & 0.25 & -2.00 & $0.50-1.00$ & $0.50-1.50$ & $1.50-2.25$ & $1.50-3.00$ & 1.18 \\
\hline February & 0.25 & -2.00 & $0.50-1.00$ & $0.50-1.50$ & $1.50-2.00$ & $1.50-3.00$ & 1.16 \\
\hline March & 0.25 & -0.50 & $0.50-1.00$ & $0.50-1.50$ & $1.50-2.00$ & $0.00-3.00$ & 1.04 \\
\hline April & 0.25 & -2.00 & $0.50-1.00$ & $0.50-1.50$ & $1.50-2.25$ & $1.50-3.00$ & 1.17 \\
\hline May & 0.25 & -2.00 & $0.50-1.00$ & $0.50-1.50$ & $1.50-2.25$ & $1.50-3.00$ & 1.17 \\
\hline June & 0.25 & -0.50 & $0.50-1.00$ & $0.50-1.50$ & $1.50-2$ & $0.00-3.00$ & 1.05 \\
\hline July & 0.25 & -2.00 & $0.50-1.00$ & $0.50-1.50$ & $1.50-2.25$ & $1.50-3.00$ & 1.18 \\
\hline August & 0.25 & -2.00 & $0.50-1.00$ & $0.50-1.50$ & $1.50-2.25$ & $1.50-3.00$ & 1.20 \\
\hline September & 0.25 & -0.50 & $0.50-0.75$ & $0.50-1.00$ & $1.25-1.50$ & $0.00-1.75$ & 0.76 \\
\hline October & 0.25 & -2.00 & $0.50-0.75$ & $0.50-1.00$ & $0.50-1.50$ & $1.50-1.75$ & 0.92 \\
\hline November & 0.25 & -2.00 & $0.25-1.00$ & $0.50-1.25$ & $0.50-1.50$ & $1.25-2.00$ & 0.95 \\
\hline December & 0.25 & -0.50 & $0.25-1.00$ & $0.50-1.25$ & $0.50-1.50$ & $0.00-2.00$ & 0.72 \\
\hline \multicolumn{8}{|l|}{2005} \\
\hline January & 0.25 & -2.00 & $0.25-1.00$ & $0.50-1.25$ & $0.50-1.50$ & $1.25-2.00$ & 0.94 \\
\hline February & 0.25 & -2.00 & $0.25-0.75$ & $0.25-1.00$ & $0.25-1.50$ & $0.75-1.50$ & 0.82 \\
\hline March & 0.25 & -2.00 & $0.25-1.00$ & $0.25-1.25$ & $0.25-1.50$ & $0.00-2.25$ & 0.95 \\
\hline April & 0.25 & -2.00 & $0.25-1.00$ & $0.25-1.25$ & $0.25-1.50$ & $0.50-2.25$ & 0.76 \\
\hline May & 0.25 & -2.00 & $0.25-1.00$ & $0.25-1.25$ & $0.25-1.50$ & $0.50-2.25$ & 0.83 \\
\hline June & 0.25 & -2.00 & $0.25-1.00$ & $0.25-1.25$ & $0.25-1.50$ & $0.00-2.25$ & 0.95 \\
\hline July & 0.25 & -2.00 & $0.25-1.00$ & $0.25-1.25$ & $0.25-1.50$ & $0.50-2.25$ & 0.72 \\
\hline August & 0.25 & -2.00 & $0.25-1.00$ & $0.25-1.25$ & $0.25-1.50$ & $0.50-2.25$ & 0.71 \\
\hline September & 0.00 & -0.20 & $0.00-0.50$ & $0.25-0.50$ & $0.25-1.50$ & $0.50-2.25$ & 0.71 \\
\hline October & 0.25 & -2.00 & $0.25-1.00$ & $0.25-1.25$ & $0.25-1.50$ & $0.50-2.25$ & 0.69 \\
\hline November & 0.25 & -2.00 & $0.25-1.00$ & $0.25-1.25$ & $0.25-1.50$ & $0.50-2.25$ & 0.69 \\
\hline December & 0.00 & -0.20 & $0.00-1.00$ & $0.25-1.25$ & $0.25-1.50$ & $0.00-2.25$ & 0.91 \\
\hline \multicolumn{8}{|l|}{2006} \\
\hline January & 0.25 & -2.00 & $0.25-1.00$ & $0.25-1.25$ & $0.25-1.50$ & $0.50-2.25$ & 0.69 \\
\hline February & 0.25 & -2.00 & $0.25-1.00$ & $0.25-1.25$ & $0.25-1.50$ & $0.50-2.25$ & 0.68 \\
\hline March & 0.25 & -2.00 & $0.25-1.00$ & $0.25-1.25$ & $0.25-1.50$ & $0.00-2.25$ & 0.94 \\
\hline April & 0.25 & -2.00 & $0.25-1.00$ & $0.25-1.25$ & $0.25-1.50$ & $0.50-2.25$ & 0.67 \\
\hline May & 0.25 & -2.00 & $0.25-1.00$ & $0.25-1.25$ & $0.50-1.50$ & $0.50-2.25$ & 0.67 \\
\hline June & 0.25 & -2.00 & $0.25-1.00$ & $0.25-1.25$ & $0.50-1.50$ & $0.00-2.25$ & 0.98 \\
\hline July & 0.25 & -2.00 & $0.25-1.00$ & $0.25-1.25$ & $0.25-1.50$ & $0.50-2.25$ & 0.66 \\
\hline August & 0.25 & -2.00 & $0.25-1.00$ & $0.25-1.25$ & $0.25-1.50$ & $0.50-2.25$ & 0.66 \\
\hline September & 0.25 & -2.00 & $0.25-1.00$ & $0.25-1.25$ & $0.50-1.50$ & $0.00-2.25$ & 0.94 \\
\hline October & 0.25 & -2.00 & $0.25-1.00$ & $0.25-1.25$ & $0.25-1.50$ & $0.50-2.25$ & 0.69 \\
\hline November & 0.25 & -2.00 & $0.25-1.00$ & $0.25-1.25$ & $0.25-1.50$ & $0.50-2.25$ & 0.76 \\
\hline December & 0.25 & -2.00 & $0.25-1.00$ & $0.25-1.25$ & $0.50-1.50$ & $0.00-2.25$ & 0.94 \\
\hline \multicolumn{8}{|l|}{2007} \\
\hline January & 0.25 & -2.00 & $0.25-1.00$ & $0.25-1.25$ & $0.25-1.50$ & $0.50-2.25$ & 0.74 \\
\hline February & 0.25 & -2.00 & $0.25-1.00$ & $0.25-1.25$ & $0.25-1.50$ & $0.50-2.25$ & 0.74 \\
\hline March & 0.25 & -2.00 & $0.25-1.00$ & $0.25-1.25$ & $0.25-1.50$ & $0.00-2.25$ & 0.94 \\
\hline April & 0.25 & -2.00 & $0.25-1.00$ & $0.25-1.25$ & $0.25-1.50$ & $0.50-2.25$ & 0.74 \\
\hline May & 0.25 & -2.00 & $0.25-1.00$ & $0.25-1.25$ & $0.25-0.75$ & $0.50-2.25$ & 0.75 \\
\hline June & 0.25 & -2.00 & $0.25-0.50$ & $0.25-0.75$ & $0.25-0.75$ & $0.50-1.40$ & 0.62 \\
\hline July & 0.25 & -2.00 & $0.25-0.50$ & $0.25-0.75$ & $0.25-0.75$ & $0.50-1.40$ & 0.62 \\
\hline August & 0.25 & -2.00 & $0.25-0.50$ & $0.25-0.75$ & $0.25-0.75$ & $0.50-1.40$ & 0.63 \\
\hline September & 0.25 & -2.00 & $0.25-0.50$ & $0.25-0.75$ & $0.25-0.75$ & $0.50-1.40$ & 0.63 \\
\hline October & 0.25 & -2.00 & $0.25-0.50$ & $0.25-0.75$ & $0.25-0.75$ & $0.50-1.40$ & 0.63 \\
\hline November & 0.25 & -2.00 & $0.25-0.50$ & $0.25-0.75$ & $0.25-0.75$ & $0.50-1.40$ & 0.65 \\
\hline December & 0.25 & -2.00 & $0.25-0.50$ & $0.25-0.75$ & $0.25-0.75$ & $0.50-1.43$ & 0.65 \\
\hline \multicolumn{8}{|l|}{2008} \\
\hline January & 0.25 & -2.00 & $0.25-0.50$ & $0.25-0.75$ & $0.25-0.75$ & $0.50-1.40$ & 0.65 \\
\hline February & 0.25 & -2.00 & $0.25-0.50$ & $0.25-0.75$ & $0.25-0.75$ & $0.50-1.40$ & 0.65 \\
\hline March & 0.25 & -2.00 & $0.25-0.50$ & $0.25-0.75$ & $0.25-0.75$ & $0.50-1.40$ & 0.65 \\
\hline
\end{tabular}

Source: Central Bank of Solomon Islands. 


\section{Table 16. Solomon Islands: Commercial Bank Interest Rates on Loans and Overdrafts, 2004-08}

(In percent, end of period)

\begin{tabular}{|c|c|c|c|c|c|c|c|}
\hline \multirow{2}{*}{$\begin{array}{l}\text { End of Period } \\
2004\end{array}$} & \multicolumn{2}{|c|}{ Personal Loans } & \multicolumn{2}{|c|}{ Other Loans } & \multicolumn{2}{|r|}{ Overdrafts } & $\begin{array}{l}\text { Weighted Average } \\
\text { Rate of Interest on } \\
\text { Loans and Overdrafts }\end{array}$ \\
\hline & 14.00 & - 17.50 & 5.90 & -21.50 & 10.00 & -20.50 & 14.69 \\
\hline 2005 & 14.00 & - 17.50 & 5.90 & -21.50 & 8.50 & -20.50 & 14.37 \\
\hline 2006 & 11.00 & -17.50 & 4.50 & -21.50 & 8.00 & -20.50 & 13.73 \\
\hline 2007 & 11.00 & - 17.50 & 4.50 & -21.50 & 8.00 & -20.50 & 14.22 \\
\hline \multicolumn{8}{|l|}{2006} \\
\hline January & 14.00 & - 17.50 & 5.90 & -21.50 & 8.50 & -20.50 & 14.39 \\
\hline February & 14.00 & -17.50 & 5.90 & -21.50 & 8.50 & -20.50 & 14.39 \\
\hline March & 14.00 & - 17.50 & 5.90 & -21.50 & 8.50 & -20.50 & 14.41 \\
\hline April & 14.00 & - 17.50 & 5.90 & -21.50 & 8.50 & -20.50 & 14.41 \\
\hline May & 14.00 & - 17.50 & 4.50 & - 21.50 & 8.00 & -20.50 & 14.01 \\
\hline June & 14.00 & - 17.50 & 4.50 & -21.50 & 8.00 & -20.50 & 14.02 \\
\hline July & 14.00 & - 17.50 & 4.50 & -21.50 & 8.00 & -20.50 & 14.00 \\
\hline August & 11.00 & -17.50 & 4.50 & -21.50 & 8.00 & -20.50 & 13.74 \\
\hline September & 11.00 & -17.50 & 4.50 & -21.50 & 8.00 & -20.50 & 13.73 \\
\hline October & 11.00 & -17.50 & 4.50 & -21.50 & 8.00 & -20.50 & 13.72 \\
\hline November & 11.00 & - 17.50 & 4.50 & -21.50 & 8.00 & -20.50 & 13.73 \\
\hline December & 11.00 & - 17.50 & 4.50 & -21.50 & 8.00 & -20.50 & 13.73 \\
\hline \multicolumn{8}{|l|}{2007} \\
\hline January & 11.00 & -17.50 & 4.50 & -21.50 & 8.00 & -20.50 & 13.72 \\
\hline February & 11.00 & -17.50 & 4.50 & -21.50 & 8.00 & -20.50 & 13.74 \\
\hline March & 11.00 & -17.50 & 4.50 & -21.50 & 8.00 & -20.50 & 13.73 \\
\hline April & 11.00 & - 17.50 & 4.50 & -21.50 & 8.00 & -20.50 & 13.72 \\
\hline May & 11.00 & - 17.50 & 4.50 & -21.50 & 8.00 & -20.50 & 13.69 \\
\hline June & 11.00 & - 17.50 & 4.50 & -21.50 & 8.00 & -20.50 & 13.74 \\
\hline July & 11.00 & -17.50 & 4.50 & -21.50 & 8.00 & -20.50 & 13.67 \\
\hline August & 11.00 & -17.50 & 4.50 & -21.50 & 8.00 & -20.50 & 14.21 \\
\hline September & 11.00 & - 17.50 & 4.50 & -21.50 & 8.00 & -20.50 & 14.21 \\
\hline October & 11.00 & -17.50 & 4.50 & -21.50 & 8.00 & -20.50 & 14.21 \\
\hline November & 11.00 & -17.50 & 4.50 & -21.50 & 8.00 & -20.50 & 14.22 \\
\hline December & 11.00 & -17.50 & 4.50 & -21.50 & 8.00 & -20.50 & 14.22 \\
\hline \multicolumn{8}{|l|}{2008} \\
\hline January & 11.00 & - 17.50 & 4.50 & - 21.50 & 8.00 & -20.50 & 14.22 \\
\hline February & 11.00 & - 17.50 & 4.50 & -21.50 & 8.00 & - 20.50 & 14.23 \\
\hline March & 11.00 & - 17.51 & 4.50 & - 21.51 & 8.00 & -20.51 & 14.23 \\
\hline
\end{tabular}

Source: Central Bank of Solomon Islands. 
Table 17. Solomon Islands: Assets and Liabilities of the National Provident Fund, 2004-08

\begin{tabular}{|c|c|c|c|c|c|c|c|c|c|c|c|c|}
\hline \multirow[b]{2}{*}{$\begin{array}{l}\text { End of } \\
\text { Period }\end{array}$} & \multicolumn{8}{|c|}{ Assets } & \multicolumn{4}{|c|}{ Liabilities } \\
\hline & $\begin{array}{l}\text { Banks } \\
\text { Term } \\
\text { Deposits }\end{array}$ & $\begin{array}{l}\text { Treasury } \\
\text { Bills }\end{array}$ & $\begin{array}{l}\text { Develop- } \\
\text { ment } \\
\text { Bonds }\end{array}$ & $\begin{array}{l}\text { Restruc- } \\
\text { tured } \\
\text { Bonds }\end{array}$ & $\begin{array}{c}\text { Loans to } \\
\text { Statutory } \\
\text { Authorities } 1 /\end{array}$ & $\begin{array}{l}\text { Loans to } \\
\text { Private } \\
\text { Sector } 2 /\end{array}$ & $\begin{array}{l}\text { Fixed } \\
\text { Assets } \\
\text { (Net) } 3 /\end{array}$ & $\begin{array}{l}\text { Other } \\
\text { Assets }\end{array}$ & $\begin{array}{l}\text { Contri- } \\
\text { bution } \\
\text { Account }\end{array}$ & $\begin{array}{l}\text { Accumu- } \\
\text { lated } \\
\text { Funds }\end{array}$ & $\begin{array}{l}\text { Other } \\
\text { Liabi- } \\
\text { lities }\end{array}$ & $\begin{array}{l}\text { Total } \\
\text { Assets and } \\
\text { Liabilities }\end{array}$ \\
\hline 2004 & 73.3 & 0.0 & 74.2 & 51.9 & 71.3 & 3.7 & 58.2 & 142.0 & 401.1 & 18.9 & 54.6 & 474.7 \\
\hline 2005 & 131.5 & 1.1 & 70.4 & 49.8 & 61.2 & 3.8 & 21.2 & 185.9 & 459.2 & 19.6 & 46.2 & 525.0 \\
\hline 2006 & 184.5 & 4.3 & 66.9 & 48.3 & 56.3 & 3.3 & 55.7 & 176.2 & 525.0 & 39.5 & 31.1 & 595.6 \\
\hline 2007 & 247.3 & 6.9 & 63.3 & 44.3 & 28.4 & 3.1 & 56.3 & 307.2 & 668.8 & 42.3 & 45.8 & 756.9 \\
\hline \multicolumn{13}{|l|}{2006} \\
\hline January & 131.4 & 1.0 & 70.1 & 49.9 & 62.6 & 38.2 & 21.2 & 157.0 & 462.0 & 21.0 & 48.4 & 531.4 \\
\hline February & 130.7 & 1.0 & 69.8 & 50.0 & 62.0 & 38.4 & 21.1 & 160.6 & 464.3 & 21.2 & 48.1 & 533.5 \\
\hline March & 129.2 & 3.4 & 69.5 & 49.8 & 61.5 & 38.6 & 21.3 & 166.7 & 467.5 & 24.7 & 47.6 & 539.8 \\
\hline April & 159.2 & 4.4 & 69.2 & 49.3 & 60.6 & 21.7 & 21.4 & 161.7 & 471.9 & 26.6 & 48.9 & 547.5 \\
\hline May & 164.2 & 5.6 & 68.9 & 48.8 & 59.2 & 21.6 & 22.0 & 168.7 & 476.6 & 39.4 & 42.8 & 558.8 \\
\hline June & 169.3 & 5.7 & 68.6 & 48.5 & 59.9 & 21.6 & 22.2 & 166.7 & 489.3 & 31.7 & 41.5 & 562.5 \\
\hline July & 182.8 & 3.3 & 68.7 & 48.2 & 57.9 & 21.8 & 21.3 & 160.1 & 493.5 & 21.8 & 48.7 & 564.0 \\
\hline August & 185.3 & 1.8 & 68.1 & 48.3 & 57.7 & 21.8 & 22.5 & 161.5 & 497.8 & 20.3 & 48.8 & 566.9 \\
\hline September & 185.3 & 0.8 & 67.6 & 48.0 & 57.3 & 21.9 & 24.8 & 170.7 & 503.8 & 23.6 & 49.0 & 576.5 \\
\hline October & 185.3 & 2.8 & 67.5 & 48.2 & 57.0 & 21.8 & 55.3 & 143.1 & 509.5 & 22.6 & 48.9 & 581.0 \\
\hline November & 172.0 & 4.3 & 67.2 & 48.2 & 56.4 & 21.7 & 55.4 & 159.0 & 520.1 & 34.0 & 30.3 & 584.3 \\
\hline December & 184.5 & 4.3 & 66.9 & 48.3 & 56.3 & 3.3 & 55.7 & 176.2 & 525.0 & 39.5 & 31.1 & 595.6 \\
\hline \multicolumn{13}{|l|}{2007} \\
\hline January & 195.1 & 1.4 & 66.5 & 47.5 & 55.8 & 3.2 & 55.7 & 174.6 & 530.8 & 38.1 & 30.9 & 599.8 \\
\hline February & 195.1 & 2.4 & 66.2 & 47.6 & 54.8 & 3.2 & 56.0 & 174.4 & 532.2 & 36.7 & 30.7 & 599.6 \\
\hline March & 210.1 & 2.8 & 66.0 & 46.6 & 55.1 & 3.3 & 57.6 & 166.4 & 540.8 & 34.9 & 32.3 & 608.0 \\
\hline April & 210.2 & 4.5 & 65.7 & 46.7 & 57.9 & 3.3 & 57.6 & 175.3 & 544.6 & 41.6 & 34.9 & 621.1 \\
\hline May & 230.7 & 5.9 & 65.4 & 46.9 & 34.3 & 3.3 & 58.4 & 193.1 & 552.0 & 61.1 & 24.8 & 637.8 \\
\hline June & 232.3 & 6.7 & 65.1 & 45.6 & 32.4 & 3.3 & 26.2 & 281.2 & 617.7 & 60.8 & 14.3 & 692.8 \\
\hline July & 230.4 & 4.3 & 65.1 & 45.3 & 31.9 & 3.2 & 26.4 & 289.2 & 622.9 & 21.1 & 51.8 & 695.8 \\
\hline August & 281.8 & 6.5 & 64.9 & 45.1 & 31.2 & 3.2 & 26.6 & 248.8 & 631.9 & 25.4 & 50.8 & 708.1 \\
\hline September & 271.1 & 2.4 & 64.6 & 44.6 & 30.2 & 3.2 & 26.6 & 272.2 & 639.7 & 24.9 & 50.3 & 715.0 \\
\hline October & 264.4 & 4.7 & 63.9 & 44.8 & 29.4 & 3.2 & 56.4 & 262.9 & 649.3 & 30.3 & 50.1 & 729.7 \\
\hline November & 260.6 & 5.8 & 63.6 & 44.6 & 28.7 & 3.2 & 56.2 & 282.7 & 659.0 & 36.5 & 49.8 & 745.3 \\
\hline December & 247.3 & 6.9 & 63.3 & 44.3 & 28.4 & 3.1 & 56.3 & 307.2 & 668.8 & 42.3 & 45.8 & 756.9 \\
\hline \multicolumn{13}{|l|}{2008} \\
\hline January & 268.4 & 7.3 & 63.0 & 44.1 & 28.1 & 3.1 & 56.2 & 288.4 & 676.7 & 36.9 & 45.0 & 758.6 \\
\hline February & 268.3 & 4.7 & 62.7 & 43.9 & 27.7 & 3.1 & 56.2 & 294.1 & 684.1 & 32.1 & 44.5 & 760.7 \\
\hline March & 288.4 & 5.5 & 62.4 & 44.0 & 25.7 & 3.1 & 56.4 & 280.2 & 696.5 & 25.1 & 44.2 & 765.8 \\
\hline
\end{tabular}

Source: National Provident Fund.

$1 /$ Includes loans to members.

2/ Includes staff loans, Western Province Housing Scheme, and NPF Housing Scheme.

$3 /$ Includes cash at bank, accrued interest, rent in arrears, and bank overdrafts. 
Table 18. Solomon Islands: Income Statement of the National Provident Fund, 2003-07 1/

(In millions of Solomon Islands dollars)

\begin{tabular}{|c|c|c|c|c|c|}
\hline & 2003 & 2004 & 2005 & 2006 & 2007 \\
\hline Interest income & 22.4 & 12.3 & 11.6 & 15.7 & $\ldots$ \\
\hline Noninterest income & 5.9 & 13.2 & 16.1 & 30.1 & $\ldots$ \\
\hline Dividends received & 0.9 & 7.1 & 7.8 & 7.7 & $\ldots$ \\
\hline Rentals & 3.9 & 4.6 & 5.0 & 5.7 & $\ldots$ \\
\hline Surcharges & 0.9 & 1.3 & 1.8 & 2 & $\ldots$ \\
\hline Sundry income & 0.1 & 0.1 & 1.4 & 10.2 & $\ldots$ \\
\hline Profit on sales of assets & 0.1 & 0.1 & 0.1 & 4.5 & $\ldots$ \\
\hline Operating income & 28.3 & 25.5 & 27.7 & 45.8 & $\ldots$ \\
\hline Less: Operating expenses & 18.1 & 67.7 & 19.9 & 22.1 & $\begin{array}{l}\cdots \\
\ldots\end{array}$ \\
\hline Administration & 17.8 & 67.5 & 19.6 & 21.8 & $\ldots$ \\
\hline Members & 0.3 & 0.2 & 0.3 & 0.3 & $\ldots$ \\
\hline Net operating income & 10.2 & -42.2 & 7.8 & 23.7 & $\ldots$ \\
\hline Interest appropriated to members & 6.8 & 7.8 & 8.9 & 15.9 & $\ldots$ \\
\hline Transfer to reserves & 3.4 & -50.1 & -1.1 & 7.7 & $\ldots$ \\
\hline
\end{tabular}

Sources: Central Bank of Solomon Islands; and NPF Annual Audited Accounts.

1/ Financial year ending in June. 
Table 19. Solomon Islands: Assets and Liabilities of the Development Bank of Solomon Islands, 2003-07

(In millions of Solomon Islands dollars)

\begin{tabular}{|c|c|c|c|c|c|c|c|c|c|c|c|c|}
\hline \multirow[b]{2}{*}{ End of Period } & \multicolumn{6}{|c|}{ Assets } & \multicolumn{5}{|c|}{ Liabilities } & \multirow[b]{2}{*}{$\begin{array}{l}\text { Total Assets } \\
\text { and Liabilities }\end{array}$} \\
\hline & $\begin{array}{r}\text { Fixed } \\
\text { Deposits }\end{array}$ & $\begin{array}{l}\text { Term } \\
\text { Loans }\end{array}$ & $\begin{array}{r}\text { Equity } \\
\text { Holdings }\end{array}$ & $\begin{array}{l}\text { Staff } \\
\text { Loans }\end{array}$ & $\begin{array}{l}\text { Fixed } \\
\text { Assets 1/ }\end{array}$ & $\begin{array}{l}\text { Other } \\
\text { Assets 2/ }\end{array}$ & S.I.G & $\begin{array}{l}\text { Liabilities } \\
\text { Overseas 3/ }\end{array}$ & N.P.F. & $\begin{array}{l}\text { Capital \& } \\
\text { Reserves }\end{array}$ & $\begin{array}{r}\text { Other } \\
\text { Liabilities }\end{array}$ & \\
\hline 2003 & 0.7 & 57.5 & 0.1 & 3.6 & 2.3 & -25.2 & 0.0 & 16.8 & 2.6 & -15.5 & 28.0 & 38.9 \\
\hline 2004 & 2.2 & 50.9 & 0.1 & 2.5 & & -27.3 & 0.0 & 20.2 & 3.8 & -14.5 & 19.9 & 29.4 \\
\hline 2005 & 0.2 & 36.5 & 0.0 & 0.7 & 2.1 & -21.3 & 0.0 & 21.1 & 3.6 & -13.6 & 7.1 & 18.1 \\
\hline 2006 & 1.8 & 17.3 & 0.0 & 0.0 & 1.0 & -8.2 & 0.0 & 21.1 & 3.5 & -13.9 & 1.2 & 11.9 \\
\hline 2007 & 0.2 & 8.2 & 0.0 & 0.0 & 0.6 & -1.1 & 0.0 & 21.1 & 0.0 & -14.1 & 1.0 & 7.9 \\
\hline \multicolumn{13}{|l|}{2006} \\
\hline January & 0.2 & 36.2 & 0.0 & 0.5 & 1.5 & -20.7 & 0.0 & 21.1 & 3.6 & -13.6 & 6.5 & 17.6 \\
\hline February & 0.4 & 33.9 & 0.0 & 0.5 & 1.5 & -19.2 & 0.0 & 21.1 & 3.6 & -13.4 & 5.7 & 17.0 \\
\hline March & 0.4 & 30.8 & 0.0 & 0.5 & 1.3 & -17.4 & 0.0 & 21.1 & 3.1 & -13.4 & 4.7 & 15.5 \\
\hline April & 0.4 & 30.4 & 0.0 & 0.4 & 1.3 & -17.4 & 0.0 & 21.1 & 3.6 & -13.3 & 3.7 & 15.0 \\
\hline May & 0.4 & 30.1 & 0.0 & 0.4 & 1.4 & -17.4 & 0.0 & 21.1 & 3.6 & -13.2 & 3.5 & 14.9 \\
\hline June & 0.4 & 2.8 & 0.0 & 0.4 & 1.4 & -16.6 & 0.0 & 21.1 & 3.6 & -13.3 & 2.6 & 13.9 \\
\hline July & 0.4 & 26.5 & 0.0 & 0.2 & 1.4 & -16.0 & 0.0 & 21.1 & 3.6 & -13.3 & 1.3 & 12.6 \\
\hline August & 0.8 & 20.0 & 0.0 & 0.2 & 1.3 & -9.7 & 0.0 & 21.1 & 3.6 & -13.3 & 1.3 & 12.7 \\
\hline September & 1.2 & 19.9 & 0.0 & 0.2 & 1.2 & -9.9 & 0.0 & 21.1 & 3.5 & -13.5 & 1.3 & 12.4 \\
\hline October & 1.5 & 19.2 & 0.0 & 0.1 & 1.0 & -9.5 & 0.0 & 21.1 & 3.5 & -13.6 & 1.3 & 12.3 \\
\hline November & 1.5 & 18.0 & 0.0 & 0.1 & 1.0 & -8.6 & 0.0 & 21.1 & 3.5 & -13.7 & 1.2 & 12.1 \\
\hline December & 1.8 & 17.3 & 0.0 & 0.0 & 1.0 & -8.2 & 0.0 & 21.1 & 3.5 & -13.9 & 1.2 & 11.9 \\
\hline \multicolumn{13}{|l|}{2007} \\
\hline January & 2.3 & 15.9 & 0.0 & 0.0 & 1.0 & -7.4 & 0.0 & 21.1 & 3.5 & -14.0 & 1.2 & 11.8 \\
\hline February & 2.5 & 14.9 & 0.0 & 0.0 & 1.0 & -6.9 & 0.0 & 21.1 & 3.5 & -14.1 & 1.1 & 11.6 \\
\hline March & 2.5 & 13.1 & 0.0 & 0.0 & 0.8 & -5.5 & 0.0 & 21.1 & 3.5 & -14.4 & 0.8 & 10.9 \\
\hline April & 2.5 & 12.3 & 0.0 & 0.0 & 0.8 & -4.7 & 0.0 & 21.1 & 3.5 & -14.5 & 0.8 & 10.9 \\
\hline May & 2.5 & 10.5 & 0.0 & 0.0 & 0.9 & -3.1 & 0.0 & 21.1 & 3.5 & -14.4 & 0.6 & 10.7 \\
\hline June & 0.0 & 10.0 & 0.0 & 0.0 & 1.1 & -4.1 & 0.0 & 21.1 & 0.0 & -14.7 & 0.6 & 6.9 \\
\hline July & 0.2 & 9.9 & 0.0 & 0.0 & 1.1 & -4.2 & 0.0 & 21.1 & 0.0 & -14.7 & 0.6 & 6.9 \\
\hline August & 0.2 & 8.8 & 0.0 & 0.0 & 1.0 & -3.2 & 0.0 & 21.1 & 0.0 & -14.8 & 0.6 & 6.8 \\
\hline September & 0.2 & 8.7 & 0.0 & 0.0 & 0.6 & -2.6 & 0.0 & 21.1 & 0.0 & -14.8 & 0.7 & 6.9 \\
\hline October & 0.2 & 8.7 & 0.0 & 0.0 & 0.7 & -2.0 & 0.0 & 21.1 & 0.0 & -14.1 & 0.7 & 7.6 \\
\hline November & 0.2 & 8.5 & 0.0 & 0.0 & 0.6 & -1.6 & 0.0 & 21.1 & 0.0 & -14.1 & 0.7 & 7.6 \\
\hline December & 0.2 & 8.2 & 0.0 & 0.0 & 0.6 & -1.1 & 0.0 & 21.1 & 0.0 & -14.1 & 1.0 & 7.9 \\
\hline
\end{tabular}

Source: Central Bank of Solomon Islands.

$1 /$ Less provision for depreciation.

2/ Includes provisions for doubtful debts.

$3 /$ Intermediated by the Solomon Islands government. 
Table 20. Solomon Islands: Income Statement of the Development Bank of Solomon Islands, 2003-07

(In millions of Solomon Islands dollars)

\begin{tabular}{|c|c|c|c|c|c|}
\hline & 2003 & 2004 & 2005 & 2006 & 2007 \\
\hline Interest income & 4.1 & 4.4 & 4.1 & 2.1 & $\ldots$ \\
\hline Interest on loans & 4.1 & 3.9 & 2.3 & 1.2 & $\ldots$ \\
\hline Interest on short-term deposits & 0.0 & 0.5 & 1.8 & 0.0 & $\ldots$ \\
\hline Less: borrowing costs & 2.1 & 0.9 & 0.0 & 0.0 & $\ldots$ \\
\hline Net interest income & 2.0 & 3.5 & 4.1 & 2.1 & $\ldots$ \\
\hline Noninterest income & 0.6 & 0.5 & 1.8 & 1.0 & $\ldots$ \\
\hline Fees & 0.0 & 0.1 & 0.0 & 0.0 & $\ldots$ \\
\hline Dividends & 0.0 & 0.0 & 0.0 & 0.0 & $\ldots$ \\
\hline Rents received & 0.0 & 0.0 & 0.0 & 0.2 & $\ldots$ \\
\hline Other income & 0.6 & 0.4 & 1.8 & 0.8 & $\ldots$ \\
\hline Profit from sale of fixed assets & 0.0 & 0.0 & 0.0 & 0.0 & $\ldots$ \\
\hline Operating income after borrowing costs & 2.6 & 4.0 & 5.9 & 2.9 & $\ldots$ \\
\hline Noninterest expenses & 8.6 & 6.2 & 2.7 & 2.4 & $\ldots$ \\
\hline General operating costs & 4.2 & 5.0 & 2.4 & 2.3 & $\ldots$ \\
\hline Director fees & 0.0 & 0.0 & 0.0 & 0.0 & $\ldots$ \\
\hline Audit fees & 0.0 & 0.0 & 0.0 & 0.0 & $\ldots$ \\
\hline Depreciation & 0.0 & 0.0 & 0.0 & 0.0 & $\ldots$ \\
\hline Doubtful debt provision & 4.4 & 1.2 & 0.3 & 0.1 & $\ldots$ \\
\hline \multicolumn{6}{|l|}{ Net operating income } \\
\hline Before foreign exchange gains/losses & -5.9 & -2.6 & -1.4 & 0.0 & $\ldots$ \\
\hline Foreign exchange gains/losses & -3.4 & 0.0 & 0.0 & 0.0 & $\ldots$ \\
\hline Net profit/loss for current year & -9.4 & -2.6 & 1.4 & -0.3 & $\ldots$ \\
\hline Retained profits/losses brought forward & -24.0 & -33.3 & -35.9 & -34.5 & $\ldots$ \\
\hline Transfer from reserve & 0.0 & 0.0 & 0.0 & 0.0 & $\ldots$ \\
\hline Net profit/loss for current year & -9.4 & -2.6 & 1.4 & -0.3 & $\ldots$ \\
\hline Retained profits/losses at end-year & -33.3 & -35.9 & -34.5 & -34.9 & $\ldots$ \\
\hline
\end{tabular}

Sources: Central Bank of Solomon Islands; DBSI Onsite Examination Report and DBSI Audited Profit and Loss Accounts. 
Table 21. Solomon Islands: Balance of Payments, 2003-07

(In millions of U.S. dollars)

\begin{tabular}{|c|c|c|c|c|c|}
\hline & 2003 & 2004 & 2005 & 2006 & $\begin{array}{r}2007 \\
\text { Est. }\end{array}$ \\
\hline Current account balance & 20.9 & 61.5 & -28.7 & -18.8 & -10.8 \\
\hline In percent of GDP & 9.1 & 23.5 & -9.8 & -5.6 & -2.8 \\
\hline Trade balance for goods & 6.7 & 11.9 & -41.2 & -62.1 & -71.1 \\
\hline Exports & 74.3 & 97.1 & 102.7 & 121.4 & 168.9 \\
\hline Of which: Logs & 49.5 & 62.6 & 67.8 & 84.6 & 109.6 \\
\hline Of which: Fish & 12.4 & 17.6 & 11.5 & 19.2 & 19.8 \\
\hline Of which: Minerals & 0.1 & 0.4 & 0.2 & 0.8 & 0.9 \\
\hline Imports & -67.5 & -85.2 & -143.9 & -183.5 & -240.0 \\
\hline Of which: Food & -15.0 & -13.6 & -20.4 & -21.6 & -30.2 \\
\hline Of which: Fuel & -11.7 & -23.3 & -42.9 & -54.4 & -56.8 \\
\hline Trade balance for services & 6.0 & 7.7 & 6.9 & 23.0 & 20.5 \\
\hline Income balance & -0.9 & 3.7 & 1.6 & 4.3 & -4.2 \\
\hline Current transfers balance & 9.1 & 38.1 & 4.1 & 16.1 & 44.0 \\
\hline Capital account balance & 12.6 & 1.5 & 27.6 & 29.4 & 36.0 \\
\hline Financial account balance & -26.1 & -39.4 & 22.7 & -16.0 & -20.9 \\
\hline Direct investment balance & -1.8 & 5.7 & 18.2 & 11.5 & 33.8 \\
\hline Other investment balance & -24.3 & -45.1 & 4.6 & -27.4 & -54.7 \\
\hline Errors and omissions & 11.4 & 19.9 & -5.7 & 14.7 & 12.7 \\
\hline Overall balance & 18.8 & 43.4 & 16.0 & 9.4 & 17.0 \\
\hline \multicolumn{6}{|l|}{ Memorandum Items } \\
\hline Gross official foreign reserves & 36.3 & 79.5 & 94.6 & 103.5 & 119.8 \\
\hline In months of projected import coverage & 4.0 & 5.5 & 5.3 & 4.2 & 4.1 \\
\hline Gross external debt (percent of GDP) & 80.5 & 68.3 & 57.2 & 52.8 & 44.1 \\
\hline Private sector & 11.6 & 9.9 & 8.6 & 7.4 & 6.2 \\
\hline Public sector & 68.9 & 58.4 & 48.6 & 45.4 & 37.9 \\
\hline External debt service (percent of GDP) & 2.1 & 1.7 & 2.5 & 1.3 & 3.2 \\
\hline Principal & 1.0 & 0.7 & 0.6 & 1.0 & 2.4 \\
\hline Interest & 1.1 & 1.0 & 1.9 & 0.4 & 0.8 \\
\hline Nominal GDP & 228.9 & 262.0 & 293.2 & 334.1 & 388.3 \\
\hline
\end{tabular}

Sources: Data provided by the authorities, and Fund Staff projections. 
Table 22. Solomon Islands: Trade Indicators, 2003-07

\begin{tabular}{|c|c|c|c|c|c|}
\hline & 2003 & 2004 & 2005 & 2006 & $\begin{array}{r}\text { Est. } \\
2007\end{array}$ \\
\hline & \multicolumn{5}{|c|}{ (Percentage change) } \\
\hline Terms of trade & -16.6 & -11.2 & -2.3 & -13.3 & -9.6 \\
\hline \multicolumn{6}{|l|}{ Export indices } \\
\hline Value & 28.4 & 30.4 & 5.9 & 17.6 & 39.4 \\
\hline Volume & 19.0 & 16.9 & 5.4 & 6.6 & 17.6 \\
\hline Unit price & 9.4 & 13.5 & 0.6 & 11.0 & 21.8 \\
\hline \multicolumn{6}{|l|}{ Import indices } \\
\hline Value & 4.5 & 26.1 & 68.9 & 27.5 & 30.8 \\
\hline Volume & 2.4 & 22.8 & 65.5 & 25.0 & 26.7 \\
\hline Unit price & 2.0 & 3.3 & 3.4 & 2.6 & 4.1 \\
\hline & \multicolumn{5}{|c|}{ (In percent of GDP) } \\
\hline \multicolumn{6}{|c|}{ Current account balance } \\
\hline Including transfers & 9.1 & 23.5 & -9.8 & -5.6 & -2.8 \\
\hline Excluding transfers & 5.2 & 8.9 & -11.2 & -10.5 & -14.1 \\
\hline
\end{tabular}

Sources: Data provided by the Solomon Islands authorities; and Fund staff estimates. 
Table 23. Solomon Islands: Composition of Exports, 2003-07

(Total values in thousands of U.S. dollars; unit values in U.S. dollars; volumes in metric tons unless noted otherwise)

\begin{tabular}{|c|c|c|c|c|c|}
\hline & 2003 & 2004 & 2005 & 2006 & $\begin{array}{r}\text { Est. } \\
2007\end{array}$ \\
\hline \multicolumn{6}{|c|}{ Timber, logs (cubic meters) } \\
\hline Value & 49,480 & 62,551 & 67,752 & 84,576 & 109,604 \\
\hline Unit value $1 /$ & 67 & 60 & 61 & 75 & 76 \\
\hline Volume & 736,000 & $1,043,150$ & $1,118,000$ & $1,130,000$ & $1,446,148$ \\
\hline Unit value change & -1.8 & -10.8 & 1.1 & 23.5 & 1.3 \\
\hline \multicolumn{6}{|l|}{ Fish } \\
\hline Value & 12,373 & 17,643 & 11,543 & 19,168 & 19,785 \\
\hline Unit value $1 /$ & 441 & 647 & 484 & 653 & 933 \\
\hline Volume & 28,072 & 27,249 & 23,853 & 29,364 & 21,196 \\
\hline Unit value change & -22.1 & 46.9 & -25.3 & 34.9 & 43.0 \\
\hline \multicolumn{6}{|l|}{ Copra } \\
\hline Value & 1,042 & 3,413 & 2,180 & 1,848 & 4,805 \\
\hline Unit value $1 /$ & 70 & 156 & 83 & 87 & 172 \\
\hline Volume & 14,849 & 21,831 & 26,182 & 21,214 & 27,905 \\
\hline Unit value change & -63.0 & 122.8 & -46.7 & 4.6 & 97.6 \\
\hline \multicolumn{6}{|l|}{ Cocoa } \\
\hline Value & 7,086 & 5,400 & 8,543 & 4,132 & 9,257 \\
\hline Unit value $1 /$ & $1,544.8$ & $1,291.5$ & $1,733.6$ & $1,079.3$ & $2,070.9$ \\
\hline Volume & 4,587 & 4,181 & 4,928 & 3,829 & 4,470 \\
\hline Unit value change & 9.3 & -16.4 & 34.2 & -37.7 & 91.9 \\
\hline \multicolumn{6}{|l|}{ Palm oil } \\
\hline Value & 0 & 0 & 0 & 4,037 & 13,759 \\
\hline Unit value $1 /$ & $\ldots$ & $\ldots$ & $\ldots$ & 744 & 802 \\
\hline Volume & 0 & 0 & 0 & 5,427 & 17,152 \\
\hline Unit value change & $\cdots$ & $\cdots$ & $\cdots$ & $\ldots$ & 7.8 \\
\hline Other (value) & 4,229 & 7,740 & 12,458 & 6,788 & 10,805 \\
\hline Total exports, f.o.b. & 74,210 & 96,747 & 102,477 & 120,548 & 168,015 \\
\hline
\end{tabular}

Sources: Data provided by the Solomon Islands authorities; and Fund staff estimates.

1/ Unit values reported are implied by data on the total volume and value of exports. 
Table 24. Solomon Islands: Composition of Imports, 2001-07 1/

\begin{tabular}{|c|c|c|c|c|c|c|c|}
\hline & 2001 & 2002 & 2003 & 2004 & 2005 & 2006 & $\begin{array}{r}\text { Est. } \\
2007\end{array}$ \\
\hline & \multicolumn{7}{|c|}{ (In thousands of U.S. dollars) } \\
\hline Oil imports & 14,352 & 11,200 & 11,709 & 23,350 & 42,905 & 54,449 & 56,760 \\
\hline Food imports & 20,547 & 16,127 & 14,961 & 13,560 & 20,384 & 21,628 & 30,238 \\
\hline Beverages \& tobacco & 1,762 & 1,639 & 924 & 1,039 & 2,385 & 1,742 & 3,427 \\
\hline Plants, vehicles \& transport equipment & 10,102 & 8,513 & 8,588 & 8,874 & 13,843 & 20,964 & 27,032 \\
\hline Building \& construction materials & 4,278 & 3,531 & 7,201 & 6,467 & 7,969 & 8,285 & 12,471 \\
\hline Chemical & 2,604 & 1,706 & 1,879 & 1,684 & 1,998 & 2,113 & 4,718 \\
\hline Other imports & 28,192 & 21,937 & 22,285 & 30,226 & 54,401 & 74,326 & 105,335 \\
\hline \multirow[t]{2}{*}{ Total imports (c.i.f.) } & 81,837 & 64,654 & 67,547 & 85,199 & 143,884 & 183,508 & 239,981 \\
\hline & \multicolumn{7}{|c|}{ (In percent of total imports) } \\
\hline Oil imports & 17.5 & 17.3 & 17.3 & 27.4 & 29.8 & 29.7 & 23.7 \\
\hline Food imports & 25.1 & 24.9 & 22.1 & 15.9 & 14.2 & 11.8 & 12.6 \\
\hline Beverages and tobacco & 2.2 & 2.5 & 1.4 & 1.2 & 1.7 & 0.9 & 1.4 \\
\hline Plants, vehicles, and transport equipment & 12.3 & 13.2 & 12.7 & 10.4 & 9.6 & 11.4 & 11.3 \\
\hline Building and construction materials & 5.2 & 5.5 & 10.7 & 7.6 & 5.5 & 4.5 & 5.2 \\
\hline Chemical & 3.2 & 2.6 & 2.8 & 2.0 & 1.4 & 1.2 & 2.0 \\
\hline Other imports & 34.4 & 33.9 & 33.0 & 35.5 & 37.8 & 40.5 & 43.9 \\
\hline
\end{tabular}

Sources: Data provided by the Solomon Islands authorities; and Fund staff estimates.

1/ Figures are based on foreign exchange transactions data from the CBSI. 
Table 25. Solomon Islands: Exports by Country of Destination, 2001-07

(Percent of total)

\begin{tabular}{|c|c|c|c|c|c|c|c|}
\hline & 2001 & 2002 & 2003 & 2004 & 2005 & 2006 & $\begin{array}{r}\text { Est. } \\
2007\end{array}$ \\
\hline $\begin{array}{l}\text { Industrial countries } \\
\text { Of which: }\end{array}$ & 29.4 & 22.7 & 19.2 & 20.4 & 17.3 & 17.7 & 15.3 \\
\hline Japan & 19.7 & 19.2 & 12.9 & 9.3 & 6.4 & 8.9 & 5.7 \\
\hline European Union & 3.9 & 1.7 & 2.1 & 6.9 & 8.0 & 5.2 & 5.1 \\
\hline Australia & 1.5 & 0.8 & 2.6 & 2.1 & 1.3 & 1.3 & 1.5 \\
\hline New Zealand & 0.3 & 0.2 & 0.5 & 0.3 & 0.3 & 0.7 & 0.4 \\
\hline Other & 4.0 & 0.8 & 1.2 & 1.9 & 1.3 & 1.5 & 2.6 \\
\hline $\begin{array}{l}\text { Asia 1/ } \\
\text { Of which: }\end{array}$ & 62.0 & 69.2 & 72.9 & 72.3 & 75.6 & 74.6 & 77.7 \\
\hline Korea & 17.8 & 14.7 & 17.0 & 16.2 & 13.5 & 9.5 & 8.5 \\
\hline China & 6.2 & 16.9 & 23.5 & 26.5 & 41.7 & 48.0 & 50.7 \\
\hline Philippines & 8.5 & 9.4 & 7.9 & 4.6 & 4.6 & 4.2 & 3.8 \\
\hline Thailand & 8.6 & 6.9 & 5.9 & 15.1 & 7.1 & 4.7 & 6.5 \\
\hline Singapore & 1.1 & 3.7 & 5.3 & 2.8 & 1.5 & 1.3 & 0.5 \\
\hline Malaysia & 1.5 & 2.2 & 1.9 & 1.0 & 1.3 & 1.3 & 2.7 \\
\hline Papua New Guinea & 1.5 & 1.4 & 1.4 & 1.3 & 1.3 & 1.4 & 1.3 \\
\hline Other & 16.8 & 14.0 & 10.0 & 4.8 & 4.7 & 4.3 & 3.8 \\
\hline Other countries & 8.6 & 8.1 & 7.9 & 7.3 & 7.0 & 7.7 & 6.9 \\
\hline
\end{tabular}

Source: IMF, Direction of Trade Statistics.

1/ Excluding Japan. 
Table 26. Solomon Islands: Imports by Country of Origin, 2001-07

(Percent of total)

\begin{tabular}{|c|c|c|c|c|c|c|c|}
\hline & 2001 & 2002 & 2003 & 2004 & 2005 & 2006 & $\begin{array}{r}\text { Est. } \\
2007\end{array}$ \\
\hline $\begin{array}{l}\text { Industrial countries } \\
\text { Of which: }\end{array}$ & 47.0 & 42.8 & 39.1 & 41.1 & 39.5 & 45.0 & 40.3 \\
\hline Australia & 28.9 & 31.4 & 27.4 & 24.7 & 26.2 & 25.5 & 26.6 \\
\hline Japan & 3.6 & 2.3 & 2.8 & 3.8 & 3.8 & 7.8 & 4.7 \\
\hline New Zealand & 4.9 & 5.0 & 4.6 & 7.8 & 4.7 & 5.1 & 4.5 \\
\hline United States & 7.1 & 2.0 & 1.5 & 1.8 & 1.2 & 2.5 & 1.8 \\
\hline Other & 2.4 & 2.1 & 2.8 & 3.1 & 3.6 & 4.2 & 2.6 \\
\hline $\begin{array}{l}\text { Asia 1/ } \\
\text { Of which: }\end{array}$ & 48.1 & 51.1 & 55.2 & 52.7 & 55.1 & 49.5 & 54.5 \\
\hline Singapore & 18.4 & 19.7 & 20.7 & 23.2 & 25.7 & 23.5 & 28.7 \\
\hline China & 1.0 & 1.2 & 1.7 & 2.1 & 2.7 & 2.2 & 3.4 \\
\hline Fiji & 3.7 & 4.6 & 4.3 & 4.8 & 4.2 & 4.2 & 4.1 \\
\hline Papua New Guinea & 3.6 & 4.5 & 4.1 & 4.7 & 4.1 & 4.1 & 4.0 \\
\hline Korea & 0.5 & 0.8 & 0.7 & 0.8 & 1.3 & 0.4 & 0.4 \\
\hline Thailand & 2.7 & 0.6 & 0.9 & 1.6 & 2.7 & 2.3 & 2.0 \\
\hline Malaysia & 1.1 & 1.0 & 1.8 & 1.5 & 2.0 & 2.3 & 2.7 \\
\hline Other & 17.0 & 18.6 & 21.0 & 14.0 & 12.4 & 10.3 & 9.2 \\
\hline Other countries & 4.9 & 6.1 & 5.6 & 6.2 & 5.4 & 5.5 & 5.2 \\
\hline
\end{tabular}

Source: IMF, Direction of Trade Statistics.

1/ Excluding Japan. 
Table 27. Solomon Islands: Services, Income, and Transfers, 2001-07

(In millions of U.S. dollars)

\begin{tabular}{|c|c|c|c|c|c|c|c|}
\hline & 2001 & 2002 & 2003 & 2004 & 2005 & 2006 & $\begin{array}{r}\text { Est. } \\
2007\end{array}$ \\
\hline Services and income & -3.9 & -2.3 & 5.1 & 11.4 & 8.4 & 27.2 & 16.3 \\
\hline Nonfactor services (net) & -6.4 & -0.4 & 6.0 & 7.7 & 6.9 & 23.0 & 20.5 \\
\hline Exports & 24.7 & 16.7 & 21.2 & 30.4 & 36.3 & 53.1 & 73.3 \\
\hline Transportation & 1.2 & 1.0 & 1.0 & 0.4 & 1.2 & 1.4 & 2.0 \\
\hline Travel & 2.2 & 1.5 & 2.3 & 4.4 & 3.3 & 4.1 & 3.5 \\
\hline Communications & 0.0 & 2.8 & 0.1 & 0.1 & 1.4 & 4.1 & 4.7 \\
\hline Financial services & 7.9 & 0.1 & 0.9 & 1.2 & 0.6 & 0.5 & 0.0 \\
\hline Other & 13.4 & 11.4 & 17.0 & 24.2 & 29.8 & 43.1 & 63.0 \\
\hline Imports & 31.1 & 17.1 & 15.3 & 22.7 & 29.4 & 30.2 & 52.7 \\
\hline Transportation & 3.3 & 3.8 & 3.1 & 6.0 & 8.7 & 9.9 & 17.3 \\
\hline Travel & 6.5 & 4.3 & 4.4 & 4.4 & 4.7 & 4.1 & 7.7 \\
\hline Communications & 1.6 & 1.8 & 2.2 & 1.6 & 1.9 & 1.7 & 2.0 \\
\hline Financial services & 6.2 & 1.6 & 0.8 & 1.6 & 1.9 & 2.9 & 5.3 \\
\hline Other & 13.5 & 5.6 & 4.6 & 9.1 & 12.2 & 11.7 & 20.5 \\
\hline Factor income (net) & 2.5 & -1.9 & -0.9 & 3.7 & 1.6 & 4.3 & -4.2 \\
\hline Credit & 6.2 & 2.7 & 3.5 & 8.5 & 8.5 & 11.4 & 10.5 \\
\hline Official interest & 1.1 & 0.5 & 0.7 & 2.5 & 3.1 & 4.4 & 6.8 \\
\hline Other & 5.1 & 2.2 & 2.8 & 6.0 & 5.3 & 7.0 & 3.6 \\
\hline Debit & 3.7 & 4.6 & 4.4 & 4.8 & 6.9 & 7.2 & 14.7 \\
\hline Official interest (accrual) & 0.5 & 0.3 & 0.6 & 0.9 & 1.0 & 0.3 & 3.1 \\
\hline Other & 3.2 & 4.3 & 3.8 & 3.9 & 5.9 & 6.8 & 11.5 \\
\hline Current transfers (net) & 12.8 & -5.5 & 9.1 & 38.1 & 4.1 & 16.1 & 44.0 \\
\hline Private sector & 4.6 & -11.0 & 0.0 & 13.8 & -0.6 & 10.4 & 2.9 \\
\hline Receipts & 24.0 & 15.9 & 18.4 & 37.1 & 35.0 & 50.2 & 39.3 \\
\hline Payments & 19.3 & 27.0 & 18.4 & 23.3 & 35.6 & 39.7 & 36.4 \\
\hline Public sector & 8.2 & 5.6 & 9.1 & 24.3 & 4.7 & 5.7 & 41.1 \\
\hline Receipts & 10.0 & 7.3 & 10.6 & 26.3 & 7.1 & 7.3 & 42.8 \\
\hline Payments & 1.9 & 1.7 & 1.6 & 2.0 & 2.4 & 1.6 & 1.7 \\
\hline
\end{tabular}

Sources: Data provided by the Solomon Islands authorities; and Fund staff estimates. 
Table 28. Solomon Islands: Medium- and Long-Term Government External Debt and Disbursements, 2001-07

(In millions of U.S. dollars)

\begin{tabular}{|c|c|c|c|c|c|c|c|}
\hline & 2001 & 2002 & 2003 & 2004 & 2005 & 2006 & $\begin{array}{r}\text { Est. } \\
2007\end{array}$ \\
\hline Total debt outstanding at year-end $1 /$ & 134.3 & 151.6 & 160.8 & 160.4 & 163.5 & 169.5 & 154.0 \\
\hline Total multilateral & 87.9 & 89.8 & 96.7 & 100.1 & 94.0 & 100.8 & 105.8 \\
\hline Asian Development Bank & 44.7 & 45.2 & 48.1 & 49.5 & 47.2 & 52.2 & 56.3 \\
\hline International Development Association & 38.5 & 40.0 & 43.9 & 46.4 & 43.2 & 45.1 & 46.1 \\
\hline OPEC & 1.9 & 1.8 & 1.6 & 1.1 & 1.0 & 0.8 & 0.6 \\
\hline International Fund for Agricultural Development & 2.8 & 2.9 & 3.1 & 3.1 & 2.7 & 2.8 & 2.8 \\
\hline Total bilateral & 30.5 & 42.3 & 41.9 & 37.0 & 35.2 & 33.9 & 32.7 \\
\hline European Community & 5.8 & 6.2 & 7.3 & 5.1 & 6.0 & 6.4 & 6.6 \\
\hline European Investment Bank & 3.0 & 3.3 & 3.9 & 2.4 & 1.4 & 1.4 & 1.4 \\
\hline United Kingdom & 2.0 & 1.7 & 0.0 & 0.0 & 0.0 & 0.0 & 0.0 \\
\hline Kuwait & 5.8 & 5.3 & 4.9 & 4.4 & 3.9 & 3.6 & 3.3 \\
\hline CDC & 0.9 & 0.8 & 0.7 & 0.6 & 0.5 & 0.5 & 0.4 \\
\hline Taiwan Province of China & 13.0 & 25.0 & 25.0 & 24.4 & 23.3 & 22.2 & 21.0 \\
\hline Commercial creditors & 8.8 & 8.4 & 8.4 & 7.4 & 6.5 & 6.3 & 0.6 \\
\hline Of which: & & & & & & & \\
\hline EFIC (Australia) & 7.3 & 7.4 & 7.8 & 6.9 & 5.8 & 5.4 & 0.0 \\
\hline Klockner (Germany) & 0.0 & 0.0 & 0.0 & 0.0 & 0.0 & 0.0 & 0.0 \\
\hline Marubeni (Hong Kong SAR) & 1.5 & 1.1 & 0.6 & 0.2 & 0.0 & 0.0 & 0.0 \\
\hline Multichem (New Zealand) & 0.0 & 0.0 & 0.0 & 0.3 & 0.3 & 0.3 & 0.2 \\
\hline Mark Barnes (USA) & 0.0 & 0.0 & 0.0 & 0.0 & 0.1 & 0.1 & 0.0 \\
\hline McConnel Dowell (New Zealand) & 0.0 & 0.0 & 0.0 & 0.0 & 0.3 & 0.6 & 0.3 \\
\hline Arrears & 7.1 & 11.1 & 13.8 & 15.8 & 27.8 & 28.4 & 15.0 \\
\hline Total disbursements & 13.0 & 12.0 & 3.1 & 0.3 & 6.0 & 6.9 & 8.2 \\
\hline Multilateral & 0.0 & 0.0 & 3.1 & 0.3 & 6.0 & 5.3 & 8.2 \\
\hline Asian Development Bank & 0.0 & 0.0 & 0.0 & 0.0 & 2.7 & 4.1 & 3.8 \\
\hline International Development Association & 0.0 & 0.0 & 3.1 & 0.3 & 3.3 & 1.2 & 4.5 \\
\hline OPEC & 0.0 & 0.0 & 0.0 & 0.0 & 0.0 & 0.0 & 0.0 \\
\hline International Fund for Agricultural Development & 0.0 & 0.0 & 0.0 & 0.0 & 0.0 & 0.0 & 0.0 \\
\hline Bilateral & 13.0 & 12.0 & 0.0 & 0.0 & 0.0 & 1.1 & 0.0 \\
\hline European Community & 0.0 & 0.0 & 0.0 & 0.0 & 0.0 & 0.0 & 0.0 \\
\hline European Investment Bank & 0.0 & 0.0 & 0.0 & 0.0 & 0.0 & 0.0 & 0.0 \\
\hline United Kingdom & 0.0 & 0.0 & 0.0 & 0.0 & 0.0 & 0.0 & 0.0 \\
\hline Kuwait & 0.0 & 0.0 & 0.0 & 0.0 & 0.0 & 0.0 & 0.0 \\
\hline CDC & 0.0 & 0.0 & 0.0 & 0.0 & 0.0 & 0.0 & 0.0 \\
\hline Taiwan Province of China & 13.0 & 12.0 & 0.0 & 0.0 & 0.0 & 1.1 & 0.0 \\
\hline Commercial creditors & 0.0 & 0.0 & 0.0 & 0.0 & 0.0 & 0.5 & 0.0 \\
\hline EFIC (Australia) & 0.0 & 0.0 & 0.0 & 0.0 & 0.0 & 0.0 & 0.0 \\
\hline Klockner (Germany) & 0.0 & 0.0 & 0.0 & 0.0 & 0.0 & 0.0 & 0.0 \\
\hline Marubeni (Hong Kong SAR) & 0.0 & 0.0 & 0.0 & 0.0 & 0.0 & 0.0 & 0.0 \\
\hline Others & $\ldots$ & $\ldots$ & $\ldots$ & $\ldots$ & $\ldots$ & 0.5 & 0.0 \\
\hline
\end{tabular}

Sources: Data provided by the Solomon Islands authorities; and Fund staff estimates.

$1 /$ Includes arrears. 
Table 29. Solomon Islands: Medium- and Long-Term External Government Debt-Service Payments, 2001-07

(Accrual basis; in millions of U.S. dollars)

\begin{tabular}{|c|c|c|c|c|c|c|c|}
\hline & 2001 & 2002 & 2003 & 2004 & 2005 & 2006 & $\begin{array}{r}\text { Est. } \\
2007\end{array}$ \\
\hline Amortization & 2.9 & 3.3 & 3.6 & 4.3 & 7.6 & 5.4 & 9.2 \\
\hline Total multilateral & 1.3 & 1.4 & 2.1 & 2.4 & 2.2 & 2.4 & 2.5 \\
\hline Asian Development Bank & 1.0 & 1.0 & 1.3 & 1.3 & 1.1 & 1.3 & 1.5 \\
\hline International Development Association & 0.2 & 0.3 & 0.6 & 0.8 & 0.8 & 0.9 & 1.0 \\
\hline OPEC & 0.0 & 0.0 & 0.2 & 0.2 & 0.2 & 0.2 & 0.0 \\
\hline International Fund for Agricultural Development & 0.1 & 0.1 & 0.1 & 0.1 & 0.2 & 0.1 & 0.0 \\
\hline Total bilateral & 1.2 & 1.5 & 1.1 & 1.5 & 2.3 & 2.0 & 4.2 \\
\hline European Community & 0.1 & 0.3 & 0.4 & 0.4 & 0.2 & 0.5 & 0.0 \\
\hline European Investment Bank & 0.1 & 0.1 & 0.1 & 0.1 & 0.3 & 0.4 & 0.0 \\
\hline United Kingdom & 0.4 & 0.4 & 0.0 & 0.0 & 0.0 & 0.0 & 0.0 \\
\hline Kuwait & 0.6 & 0.6 & 0.6 & 0.4 & 0.6 & 0.4 & 3.0 \\
\hline CDC & 0.0 & 0.1 & 0.1 & 0.1 & 0.1 & 0.1 & 0.1 \\
\hline Taiwan Province of China & 0.0 & 0.0 & 0.0 & 0.6 & 1.1 & 0.6 & 1.1 \\
\hline Commercial creditors & 0.4 & 0.4 & 0.4 & 0.4 & 3.2 & 1.0 & 2.5 \\
\hline EFIC (Australia) & 0.0 & 0.0 & 0.0 & 0.0 & 0.5 & 0.5 & 2.1 \\
\hline Klockner (Germany) & 0.0 & 0.0 & 0.0 & 0.0 & 0.0 & 0.0 & 0.0 \\
\hline Marubeni (Hong Kong SAR) & 0.4 & 0.4 & 0.4 & 0.4 & 2.6 & 0.0 & 0.0 \\
\hline Multichem (New Zealand) & 0.0 & 0.0 & 0.0 & 0.0 & 0.0 & 0.0 & 0.1 \\
\hline Mark Barnes (USA) & 0.0 & 0.0 & 0.0 & 0.0 & 0.0 & 0.0 & 0.0 \\
\hline McConnel Dowell (New Zealand) & 0.0 & 0.0 & 0.0 & 0.0 & 0.0 & 0.4 & 0.3 \\
\hline Interest payments & 2.2 & 2.5 & 2.7 & 2.6 & 2.5 & 2.1 & 3.3 \\
\hline Total multilateral & 0.8 & 0.8 & 1.0 & 1.0 & 1.0 & 0.9 & 0.9 \\
\hline Asian Development Bank & 0.4 & 0.5 & 0.6 & 0.6 & 0.5 & 0.5 & 0.5 \\
\hline International Development Association & 0.3 & 0.3 & 0.3 & 0.3 & 0.3 & 0.3 & 0.3 \\
\hline OPEC & 0.1 & 0.1 & 0.0 & 0.0 & 0.1 & 0.1 & 0.0 \\
\hline International Fund for Agricultural Development & 0.0 & 0.0 & 0.0 & 0.0 & 0.0 & 0.0 & 0.0 \\
\hline Total bilateral & 0.9 & 1.3 & 1.3 & 1.3 & 1.3 & 1.0 & 2.4 \\
\hline European Community & 0.0 & 0.1 & 0.1 & 0.1 & 0.0 & 0.1 & 0.0 \\
\hline European Investment Bank & 0.1 & 0.1 & 0.1 & 0.1 & 0.0 & 0.0 & 0.0 \\
\hline United Kingdom & 0.1 & 0.1 & 0.0 & 0.0 & 0.0 & 0.0 & 0.0 \\
\hline Kuwait & 0.2 & 0.2 & 0.2 & 0.2 & 0.3 & 0.4 & 1.6 \\
\hline CDC & 0.0 & 0.0 & 0.0 & 0.0 & 0.0 & 0.0 & 0.0 \\
\hline Taiwan Province of China & 0.5 & 0.9 & 0.9 & 0.9 & 0.9 & 0.4 & 0.8 \\
\hline Commercial creditors & 0.5 & 0.4 & 0.4 & 0.4 & 0.2 & 0.2 & 0.0 \\
\hline EFIC (Australia) & 0.3 & 0.3 & 0.3 & 0.3 & 0.2 & 0.2 & 0.0 \\
\hline Klockner (Germany) & 0.0 & 0.0 & 0.0 & 0.0 & 0.0 & 0.0 & 0.0 \\
\hline Marubeni (Hong Kong SAR) & 0.1 & 0.1 & 0.0 & 0.0 & 0.0 & 0.0 & 0.0 \\
\hline Multichem (New Zealand) & 0.0 & 0.0 & 0.0 & 0.0 & 0.0 & 0.0 & 0.0 \\
\hline Mark Barnes (USA) & 0.0 & 0.0 & 0.0 & 0.0 & 0.0 & 0.0 & 0.0 \\
\hline McConnel Dowell (New Zealand) & 0.0 & 0.0 & 0.0 & 0.0 & 0.0 & 0.0 & 0.0 \\
\hline \multicolumn{8}{|l|}{ Memorandum items: } \\
\hline Arrears & 7.1 & 11.1 & 13.8 & 15.8 & 27.8 & 28.4 & 15.0 \\
\hline Principal & 4.7 & 7.4 & 7.6 & 9.0 & 24.1 & 23.9 & 11.4 \\
\hline Interest & 2.4 & 3.7 & 6.2 & 6.9 & 3.7 & 4.5 & 3.6 \\
\hline
\end{tabular}

Sources: Data provided by the Solomon Islands authorities; and Fund staff estimates. 

Table 30. Solomon Islands: Medium- and Long-Term
External Debt Indicators, 2001-07

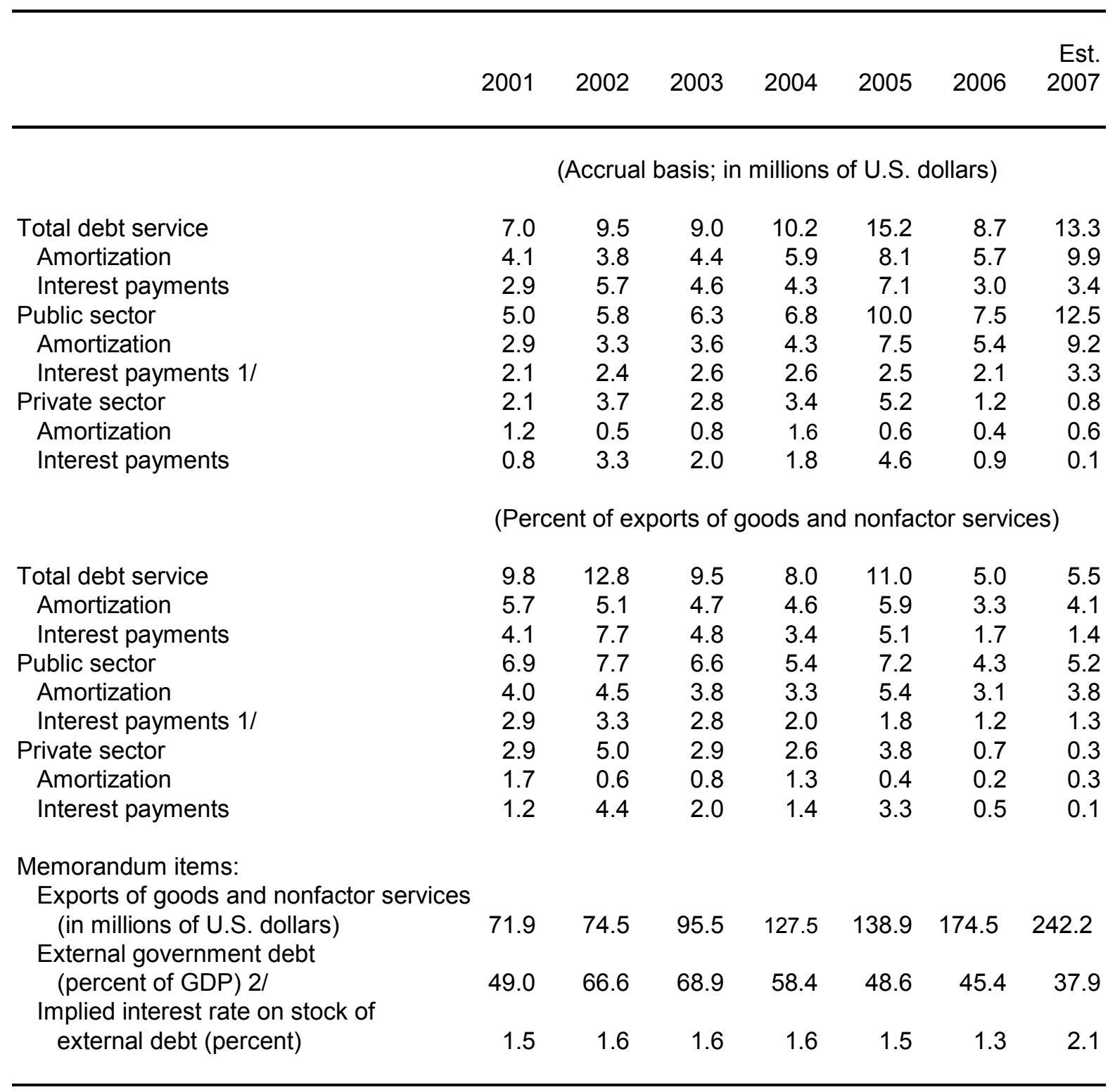

Sources: Data provided by the Solomon Islands authorities; and Fund staff estimates.

1/ Excludes interest arrears.

2/ Includes external debt arrears. 
Table 31. Solomon Islands: Bilateral Exchange Rates, 2003-08

\begin{tabular}{|c|c|c|c|c|c|c|}
\hline & $\begin{array}{r}\text { Australian } \\
\text { Dollar }\end{array}$ & $\begin{array}{l}\text { Japanese } \\
\text { Yen 1/ }\end{array}$ & $\begin{array}{r}\text { New Zealand } \\
\text { Dollar }\end{array}$ & $\begin{array}{l}\text { U.S. } \\
\text { Dollar }\end{array}$ & $\begin{array}{r}\text { U.K. Pound } \\
\text { Sterling }\end{array}$ & SDR \\
\hline \multicolumn{7}{|c|}{ Annual averages } \\
\hline 2003 & 4.94 & 6.50 & 4.40 & 7.51 & 12.37 & 10.57 \\
\hline 2004 & 5.51 & 6.95 & 4.97 & 7.49 & 13.72 & 11.11 \\
\hline 2005 & 5.74 & 6.81 & 5.29 & 7.53 & 13.65 & 11.10 \\
\hline 2006 & 5.76 & 6.54 & 4.95 & 7.60 & 14.09 & 11.22 \\
\hline 2007 & 6.45 & 6.51 & 5.66 & 7.65 & 15.35 & 11.75 \\
\hline \multicolumn{7}{|l|}{2005} \\
\hline January & 5.81 & 7.22 & 5.33 & 7.51 & 14.11 & 11.45 \\
\hline February & 5.93 & 7.17 & 5.42 & 7.51 & 14.43 & 11.50 \\
\hline March & 5.80 & 7.00 & 5.32 & 7.51 & 14.14 & 11.35 \\
\hline April & 5.87 & 7.10 & 5.47 & 7.51 & 14.37 & 11.40 \\
\hline May & 5.68 & 6.96 & 5.35 & 7.52 & 13.67 & 11.09 \\
\hline June & 5.74 & 6.81 & 5.26 & 7.51 & 13.47 & 10.94 \\
\hline July & 5.71 & 6.71 & 5.14 & 7.52 & 13.21 & 10.92 \\
\hline August & 5.63 & 6.77 & 5.17 & 7.53 & 13.43 & 10.99 \\
\hline September & 5.76 & 6.68 & 5.21 & 7.56 & 13.35 & 10.96 \\
\hline October & 5.67 & 6.55 & 5.33 & 7.58 & 13.47 & 10.95 \\
\hline November & 5.67 & 6.33 & 5.31 & 7.58 & 13.08 & 10.79 \\
\hline December & 5.56 & 6.42 & 5.16 & 7.58 & 13.04 & 10.83 \\
\hline \multicolumn{7}{|l|}{2006} \\
\hline January & 5.70 & 6.45 & 5.17 & 7.59 & 13.44 & 10.97 \\
\hline February & 5.61 & 6.53 & 5.01 & 7.59 & 13.26 & 10.90 \\
\hline March & 5.44 & 6.47 & 4.66 & 7.59 & 13.17 & 10.94 \\
\hline April & 5.73 & 6.64 & 4.81 & 7.59 & 13.73 & 11.17 \\
\hline May & 5.81 & 6.78 & 4.86 & 7.60 & 14.30 & 11.36 \\
\hline June & 5.65 & 6.61 & 4.59 & 7.60 & 13.94 & 11.24 \\
\hline July & 5.82 & 6.62 & 4.70 & 7.60 & 14.17 & 11.28 \\
\hline August & 5.80 & 6.49 & 4.95 & 7.61 & 14.52 & 11.33 \\
\hline September & 5.70 & 6.47 & 4.98 & 7.62 & 14.24 & 11.24 \\
\hline October & 5.86 & 6.47 & 5.07 & 7.62 & 14.46 & 11.27 \\
\hline November & 5.98 & 6.54 & 5.17 & 7.62 & 14.90 & 11.48 \\
\hline December & 6.03 & 6.40 & 5.38 & 7.62 & 14.95 & 11.46 \\
\hline \multicolumn{7}{|l|}{2007} \\
\hline January & 5.90 & 6.28 & 5.25 & 7.64 & 14.92 & 11.38 \\
\hline February & 6.02 & 6.45 & 5.35 & 7.64 & 14.95 & 11.50 \\
\hline March & 6.17 & 6.49 & 5.45 & 7.64 & 14.96 & 11.54 \\
\hline April & 6.30 & 6.39 & 5.64 & 7.64 & 15.24 & 11.64 \\
\hline May & 6.30 & 6.29 & 5.58 & 7.65 & 15.12 & 11.57 \\
\hline June & 6.49 & 6.20 & 5.88 & 7.65 & 15.33 & 11.59 \\
\hline July & 6.56 & 6.43 & 5.90 & 7.65 & 15.54 & 11.72 \\
\hline August & 6.29 & 6.59 & 5.38 & 7.66 & 15.48 & 11.74 \\
\hline September & 6.77 & 6.67 & 5.78 & 7.67 & 15.58 & 11.94 \\
\hline October & 7.07 & 6.68 & 5.87 & 7.67 & 15.90 & 12.05 \\
\hline November & 6.79 & 6.95 & 5.91 & 7.66 & 15.83 & 12.19 \\
\hline December & 6.76 & 6.72 & 5.93 & 7.66 & 15.35 & 12.11 \\
\hline \multicolumn{7}{|l|}{2008} \\
\hline January & 6.81 & 7.20 & 6.01 & 7.66 & 15.28 & 12.22 \\
\hline February & 7.25 & 7.32 & 6.25 & 7.66 & 15.20 & 12.34 \\
\hline March & 7.03 & 7.66 & 6.08 & 7.66 & 15.24 & 12.60 \\
\hline April & 7.15 & 7.36 & 5.94 & 7.66 & 15.07 & $\ldots$ \\
\hline May & 7.32 & 7.25 & 5.98 & 7.66 & 15.12 & $\ldots$ \\
\hline June & 7.38 & 7.20 & 5.84 & 7.66 & 15.26 & $\ldots$ \\
\hline
\end{tabular}

Sources: IMF, International Financial Statistics; and Fund staff estimates.

$1 /$ Solomon Islands dollar per 100 yen. 
Table 32. Solomon Islands: Exchange Rate Indicators, 2003-08

\begin{tabular}{|c|c|c|c|c|c|}
\hline & & $\begin{array}{r}\text { Real Effective } \\
\text { Exchange Rate }\end{array}$ & $\begin{array}{r}\text { Nominal Effective } \\
\text { Exchange Rate }\end{array}$ & $\begin{array}{l}\text { Relative Price } \\
\text { Index 1/ }\end{array}$ & $\begin{array}{l}\text { Exchange Rate } \\
\text { Index 2/ }\end{array}$ \\
\hline \multicolumn{6}{|c|}{ Annual averages } \\
\hline 2003 & & 78.5 & 64.4 & 122.1 & 67.8 \\
\hline 2004 & & 77.1 & 60.3 & 128.1 & 68.0 \\
\hline 2005 & & 79.5 & 59.1 & 134.6 & 67.6 \\
\hline 2006 & & 85.5 & 58.5 & 146.2 & 66.9 \\
\hline 2007 & & 84.6 & 55.0 & 153.9 & 66.5 \\
\hline \multirow[t]{4}{*}{2003} & I & 80.6 & 67.1 & 120.2 & 68.0 \\
\hline & II & 79.5 & 64.8 & 122.7 & 67.7 \\
\hline & III & 78.4 & 64.2 & 122.2 & 67.7 \\
\hline & IV & 75.7 & 61.4 & 123.4 & 67.8 \\
\hline \multirow[t]{4}{*}{2004} & I & 75.3 & 59.6 & 126.5 & 67.9 \\
\hline & II & 78.5 & 61.5 & 127.7 & 68.1 \\
\hline & III & 78.5 & 61.3 & 128.3 & 68.1 \\
\hline & IV & 76.2 & 58.8 & 129.8 & 67.9 \\
\hline \multirow[t]{4}{*}{2005} & I & 75.4 & 57.9 & 130.3 & 67.8 \\
\hline & II & 77.7 & 58.7 & 132.4 & 67.7 \\
\hline & III & 82.2 & 59.6 & 138.1 & 67.6 \\
\hline & IV & 82.8 & 60.2 & 137.8 & 67.2 \\
\hline \multirow[t]{4}{*}{2006} & I & 85.2 & 59.6 & 143.0 & 67.0 \\
\hline & II & 85.4 & 58.7 & 145.5 & 67.0 \\
\hline & III & 85.6 & 58.3 & 147.0 & 66.9 \\
\hline & IV & 85.8 & 57.5 & 149.2 & 66.7 \\
\hline \multirow[t]{4}{*}{2007} & 1 & 86.6 & 56.9 & 152.2 & 66.6 \\
\hline & II & 84.3 & 55.6 & 151.7 & 66.6 \\
\hline & III & 83.4 & 54.7 & 152.4 & 66.4 \\
\hline & IV & 84.2 & 52.9 & 159.5 & 66.4 \\
\hline 2008 & I & 82.8 & 51.5 & 160.8 & 66.4 \\
\hline
\end{tabular}

Source: IMF, Information Notice System.

1/ Consumer price index in the Solomon Islands relative to a weighted average of consumer price indices in major trading partners.

2/ U.S. dollar/Solomon Islands dollar exchange rate index. 